\title{
The Nico Pérez terrane (Uruguay) and its archean and paleoproterozoic inheritance
}

\author{
*Leda Sánchez Bettucci ${ }^{1}$, Umberto Cordani ${ }^{2}$, Judith Loureiro ${ }^{3}$, Elena Peel ${ }^{1}$, Santiago Fort ${ }^{1}$, Kei Sato²
}

\author{
${ }^{1}$ Universidad de la República, Facultad de Ciencias, Iguá 4225 esquina Mataojo, Montevideo, 11400 Uruguay. \\ leda@fcien.edu.uy; sfort@fcien.edu.uy; elena@fcien.edu.uy \\ ${ }^{2}$ Instituto de Geociências-Universidade de São Paulo, R. do Lago, 562-Butantã, SP, 05508-080, Brasil. \\ ucordani@usp.br; keisato@usp.br \\ ${ }^{3}$ Dirección Nacional de Minería y Geología-MIEM Hervidero 2861, Montevideo, Uruguay. \\ Judith.Loureiro@miem.gub.uy
}

*Corresponding author: leda@fcien.edu.uy

\begin{abstract}
A U-Pb SHRIMP zircon geochronological study was carried out in the Nico Pérez Terrane in the centraleastern portion of Uruguay with the aim of constraining its geological evolution and its cratonic affinity. Nico Pérez Terrane is made up by a mosaic of tectonic blocks with diferente lithologies and sizes. This terrane is limited to the west by the Piedra Alta Terrane through the Sarandí del Yí shear zone, and to the east, is in tectonic contact through the Retamosa thrust with the Dom Feliciano Belt (Brasiliano orogenic cycle). Lithologically, the Nico Pérez Terrane is composed by medium to high grade metamorphic rock contained in three tectonic blocks (Pavas, Valentines and Rivera blocks) represented mainly by granitoids, as well as ortho and parametamorphic rocks, such as amphibolites, metapyroxenites, BIFs, schists, quartzites with fuchsite, among others. Archean inheritance, Rhyacian, Statherian, and Neoproterozoic magmatic ages are reported here. Similar ages, which show Archaean inheritance and Neoproterozoic imprint, reported in units of the Piedra Alta Terrane modify the idea that the Río de La Plata Craton only corresponds to a juvenile Paleoproterozoic tectonic unit. This, together with recently published geophysical information, supports the cratonic affinity of the Nico Pérez Terrane with the Río de La Plata Craton.
\end{abstract}

Keywords: Nico Pérez Terrane, Río de La Plata Craton, Archean inliers, U-Pb SHRIMP zircon ages.

RESUMEN. EI terreno Nico-Pérez (Uruguay) y su herencia arcaica y paleoproterozoica. Un estudio geocronológico U-Pb SHRIMP en circón fue realizado en rocas del Terreno Nico Pérez, con el objetivo de circunscribir la evolución geológica y su afinidad cratónica. Este terreno, localizado en la porción central del Uruguay, está constituído por un mosaico de tres bloques tectónicos y se encuentra limitado al oeste por el Terreno Piedra Alta a través de la zona de cizallamiento Sarandí del Yí. Al este, se encuentra en contacto tectónico a través del corrimiento Retamosa con el Cinturón Dom Feliciano (Ciclo Orogénico Brasiliano). Litológicamente, el Terreno Nico Pérez está compuesto por rocas metamórficas de grado medio a alto (bloques Pavas, Valentines y Rivera) representados principalmente por granitoides, así como por rocas orto- y parametamórficas, tales como anfibolitas, metapiroxenitas, BIFs, esquistos, cuarcitas con fucsita, entre otras. Se presenta en este trabajo un conjunto de edades magmáticas que poseen herencia arqueana, y edades riacianas, estaterianas y neoproterozoicas. Edades similares a estas, que muestran herencia arqueana e impronta neoproterozoica, reportadas en unidades del Terreno Piedra Alta, modifican la idea de que el Cratón del Río de La Plata corresponde a una unidad tectónica paleoproterozoica juvenil. Esto, junto con la información geofísica publicada recientemente, respalda la afinidad cratónica del Terreno Nico Pérez con el Cratón del Río de La Plata. 


\section{Introduction}

The Nico Pérez Terrane, located in the central portion of Uruguay (Fig. 1), records the oldest basement ages of the region. Bossi and Campal (1992) identified this terrane from the main outcropping areas of the Río de La Plata Craton (sensu Almeida et al., 1973) and based on lithological and structural data they defined it as a Paleoproterozoic block affected by tectono-magmatic events related to the Brasiliano-Panafrican orogenic cycle. Aditionally, these authors postulated that the Nico Pérez Terrane represents the foreland of the Neoproterozoic orogenic belt known as Dom Feliciano Belt (Fragoso-César, 1980). Later works by Hartmann et al. (2001), Santos et al. (2003), Mallmann et al. (2007), Gaucher
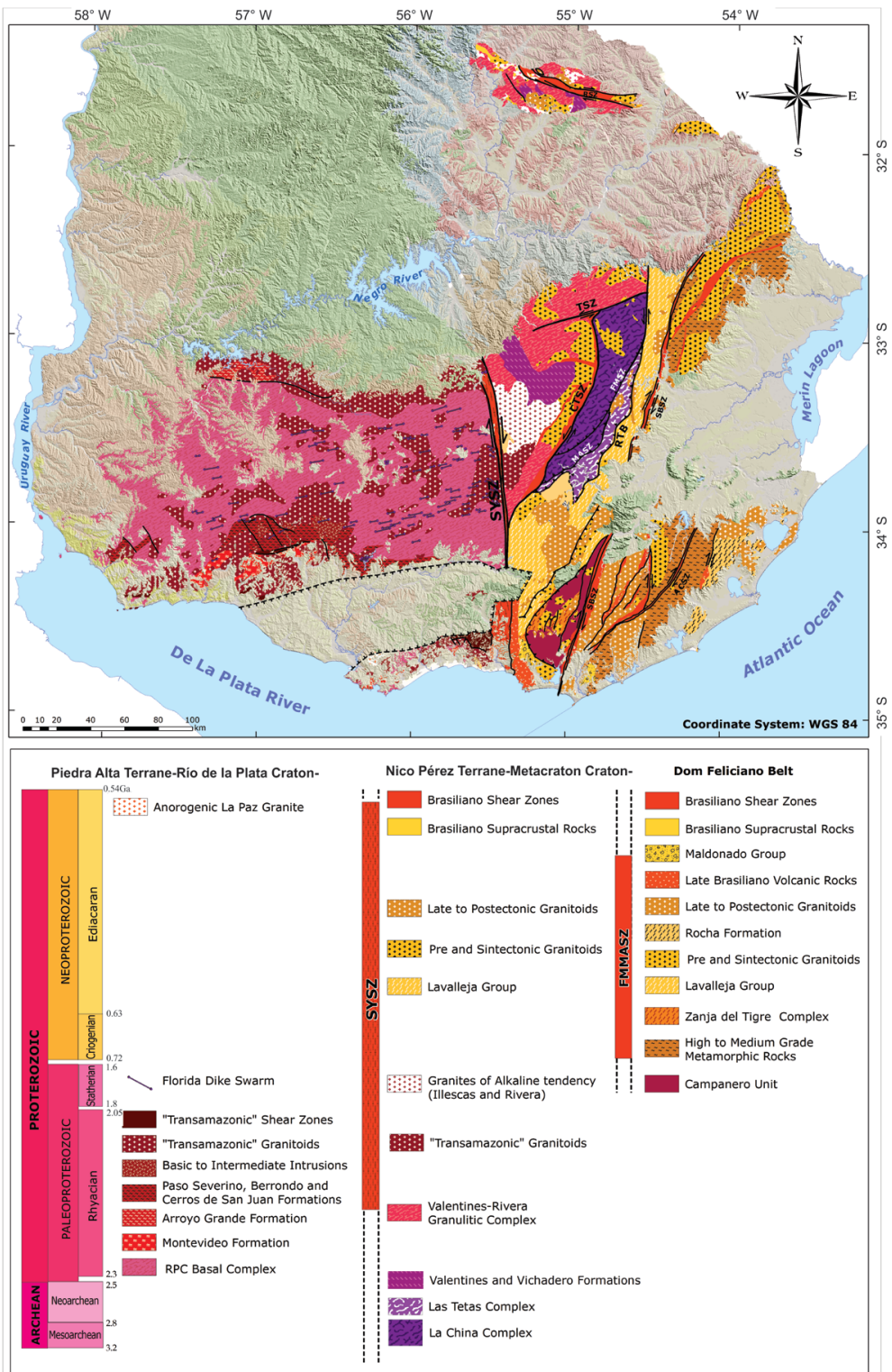

FIG. 1. Geologic map from Uruguay showing the basement most conspicuous units. SYSZ: Sarandí del Yi shear zone; FMSZ: Fraile Muerto shear zone; MASZ: María Albina shear zone; CTSZ: Cueva del Tigre shear zone; RT: Retamosa thrust; TSZ: Tupambaé shear zone; RSZ: Rivera shear zone; SBSZ: Sierra Ballena shear zone; ACSZ: Alférez-Cordillera shear zone. (Modified from Loureiro et al., 2019). 
et al. (2010a), Oyhantçabal et al. (2012, 2018) and Oriolo et al. (2016a), among others, allowed the recognition of Archean and Paleoproterozoic basement rocks and the intense impact of Neoproterozoic granitic intrusions and deformation in the Nico Pérez terrane. Additionally, several authors (e.g., Bossi et al., 1998; Hartmann et al., 2001; Bossi and Gaucher, 2004; Mallmann et al., 2007; Gaucher et al., 2010 (a, b); Oriolo et al., 2016a; Oyhantçabal et al., 2018) have extended its eastern border to the Sierra Ballena shear zone (Fig. 2). Oyhantçabal et al. (2011) challenged its affinity with the Río de la Plata Craton based on lithologic, structural, geochronologic, and isotopic data. Recently, Oriolo et al. (2016a, 2017) interpreted this terrane as a basement inlier of the Dom Feliciano Belt, and they suggested its affinity with the West Africa Congo Craton based on tectonostratigraphy, $\mathrm{U}-\mathrm{Pb}$ ages, and $\mathrm{Hf}$ isotopic data.
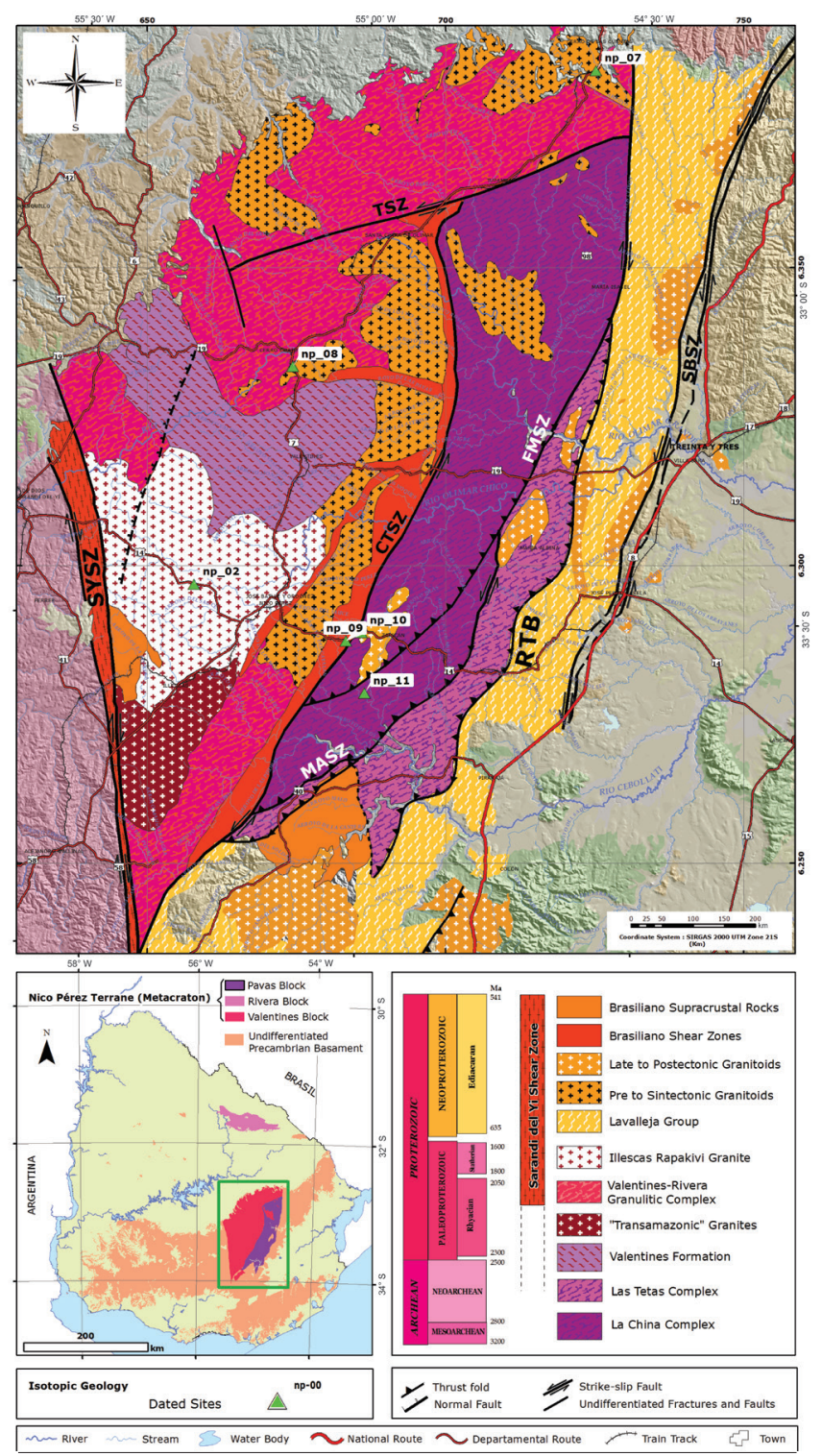

FIG 2. Geologic map of the Valentines and Pavas Blocks (Nico Pérez Terrane) showing de dated points. SYSZ: Sarandí del Yí shear zone; FMSZ: Fraile Muerto shear zone; MASZ: María Albina shear zone; CTSZ: Cueva del Tigre shear zone; RT: Retamosa thrust; TSZ: Tupambaé shear zone; SBSZ: Sierra Ballena shear zone. Modified from Loureiro et al. (2019). 
After almost 30 years since its original definition, the Nico Pérez Terrane is considered an ArcheanPaleoproterozoic crustal block with Neoproterozoic reworking related to the Brasiliano Orogeny, even though its affinity with the Río de la Plata Craton and its limits are still being subjects of controversy (e.g., Sánchez Bettucci et al., 2010a; Santos et al., 2019; Oyhantçabal et al., 2018; Bologna et al., 2019; Loureiro et al., 2019). In this paper, new SHRIMP $\mathrm{U}-\mathrm{Pb}$ zircon ages from rocks of nine samples from six sites (see Fig. 2) are presented. These ages are integrated with geological, geophysical, and geochronological evidence to provide constraints for the understanding of the geological evolution of the Uruguayan basement. Also, an appraisal about the limits and the affinity of the Nico Pérez Terrane with the Congo or Río de La Plata cratons are presented based on available information.

\section{Geological framework of the Nico Pérez Terrane}

The Nico Pérez terrane is located between the Sarandí del Yí shear zone to the west and the Retamosa thrust (RT) to the east and south (Fig. 1). Its northeastern limit is along the Fraile Muerto shear zone. Both, the Retamosa thrust and Fraile Muerto shear zone separate it from the supracrustal sequence of the Dom Feliciano Belt (e.g., Bossi and Campal, 1992; Sánchez Bettucci et al., 2010b). Meanwhile, the Sarandí del Yí shear zone separates the Nico Pérez Terrane from the Piedra Alta Terrane developed to the west. The Piedra Alta Terrane (Bossi et al., 1993) includes low to medium metamorphic orogenic belts (ca. $2.1 \mathrm{Ga}$ ), a layered mafic complex, a gneissicmigmatitic basement, pre- to syn- orogenic granitoids, mafic and felsic late to post orogenic magmatism (1.9-2.3 Ga), A-type rapakivi granites $(2.078 \mathrm{Ga})$, and extensional magmatism $(1.7 \mathrm{Ga})$ represented by a mafic dike swarm (Sánchez Bettucci et al., 2010a; Franceschinis et al., 2019 and references therein). All these lithostratigraphic units were assigned to the Transamazonian orogenic cycle (Hurley et al., 1967; Choubert and Faure-Muret, 1969; Almeida et al., 1973) or Transplatense orogenic cycle (Santos et al., 2019).

From Almeida et al. (1973) the current areas of the Nico Pérez Terrane and Piedra Alta Terrane have been considered a part of the Río de la Plata Craton. The Río de La Plata Craton (sensu Almeida et al., 1973) occupies approximately the third part of the south-western region of Uruguay (Fig. 1). It extends towards the north of the country in Rivera and Aceguá (near the Brazilian border), and crops out in Brazil, at the eastern part of Rio Grande do Sul State (Taquarembó and Encruzilhada terranes). Also, it crops out in the Tandil mountain ranges in Argentina. Oyhantçabal et al. (2011) questioned this concept in a review of the Río de La Plata Craton. These authors based on features like $\mathrm{Sm} / \mathrm{Nd} \mathrm{T}_{\mathrm{DM}}$ model ages (Piedra Alta Terrane $\mathrm{T}_{\mathrm{DM}}$ 2.8-2.3 Ga; Nico Pérez Terrane $\mathrm{T}_{\mathrm{DM}}$ 3.0-2.6 and 2.3-1.6 Ga), crystallization ages (Piedra Alta Terrane 2.2-2.1 Ga; Nico Pérez Terrane 3.1-0.57 Ga), and differences in gravity signature for both terranes, proposed that the Nico Pérez Terrane and Taquarembo block are allocthonous to the Río de La Plata Craton, comprising only the Piedra Alta and Tandilia terranes. However, Santos et al. (2017) reported for the first time Archean inheritance ( $\mathrm{T}_{\mathrm{DM}} \mathrm{Hf}: 2.52 \mathrm{Ga}$; average epsilón Hf: 3.62) and Brasiliano reworking in the Piedra Alta Terrane, modifying the established idea of only Rhyacian juvenile crust. Besides, Bologna et al. (2019) based on a magnetotelluric study of the Piedra Alta and Nico Pérez terranes mentioned that their results showed no lithospheric-scale contrast in the electrical resistivity across the Sarandí del Yí shear zone. In addition, they suggested the Nico Pérez Terrane is the remobilized metacratonic portion (sensu Liégeois et al., 2013) of the Río de La Plata Craton, as previously suggested by Santos et al. (2019). Regional syntheses of the tectonic evolution of the Río de La Plata Craton in Uruguay are available in Rapela et al. (2007); Sánchez Bettucci et al. (2010a); Oyhantçabal et al. (2011, 2018); Cingolani et al. (2012); Santos et al. (2019); Bologna et al. (2019) and references therein.

As stated above, the Nico Pérez Terrane is made up of Archean and Paleoproterozoic high-grade metamorphic sequences and granitoid rocks, as well as post-orogenic magmatic rocks. It comprises the Pavas, Valentines, Rivera and Aceguá structural blocks, as shown in figures 1 and 2. The main features of each of these structural blocks are presented below.

\subsection{Pavas Block}

The Pavas Block (sensu Preciozzi et al., 1985) is composed by La China and Las Tetas Archean complexes (Hartmann et al., 2001), covering approximately $2,025 \mathrm{~km}^{2}$ and $635 \mathrm{~km}^{2}$, respectively 
(Loureiro et al., 2019), both with an NE-SW regional structural trend. To the north and west, La China Complex is in tectonic contact with the Valentines Block through Cueva del Tigre shear zone. To the south-east this complex thrusts Las Tetas Complex by the María Albina shear zone, and to the north-east, it is in tectonic contact with the supracrustal rocks of the Dom Feliciano Belt by the Fraile Muerto shear zone (see Fig. 2).

La China Complex is described as a set of orthogneisses, granitoids, and a greenstone belt consisting of igneous mafic and ultramafic rocks with subordinate cherts, that underwent significant deformation and metamorphism under amphibolite facies conditions; also it contains a few Neoproterozoic intrusions. The complex presents a general composition of tonalite-trondhjemite-granodiorite (TTG) according to Hartmann et al. (2001). The protoliths of the metaultramafic rocks have been interpreted as hazburgites, dunites and komatiites. Serpentinites, talc-chlorite-tremolite schists and amphibolites are common (Preciozzi et al., 1979, 1985; Hartmann et al., 2001; Gaucher et al., 2014a, b). Metatonalites are the rocks where most of the Archean ages have been reported. They present irregular compositional bands, with amphibole and quartz-feldspathic rich bands (Fig. 3). The Zapicán diorite intrudes the La China Complex, and crops out as NNE-SSW body (Lossada et al., 2014). It comprises diorites to granodiorites, often containing hornblende and gabbroic enclaves (Fig. 3).

Las Tetas Complex is defined as a succession of metasedimentary rocks affected by amphibolite facies metamorphism, consisting of meta-quartzites with fuchsite, metaconglomerates, micaschists with staurolite and garnet, gneisses with muscovite and tourmaline, BIFs and dolomitic marbles (Hartmann et al., 2001; Masquelin et al., 2017; and references therein). This complex has a decreasing metamorphic grade from north to south (Gaucher et al., 2014a; Oyhantçabal et al., 2018) from quartzites with sillimanite in the north, to micaceous shales with
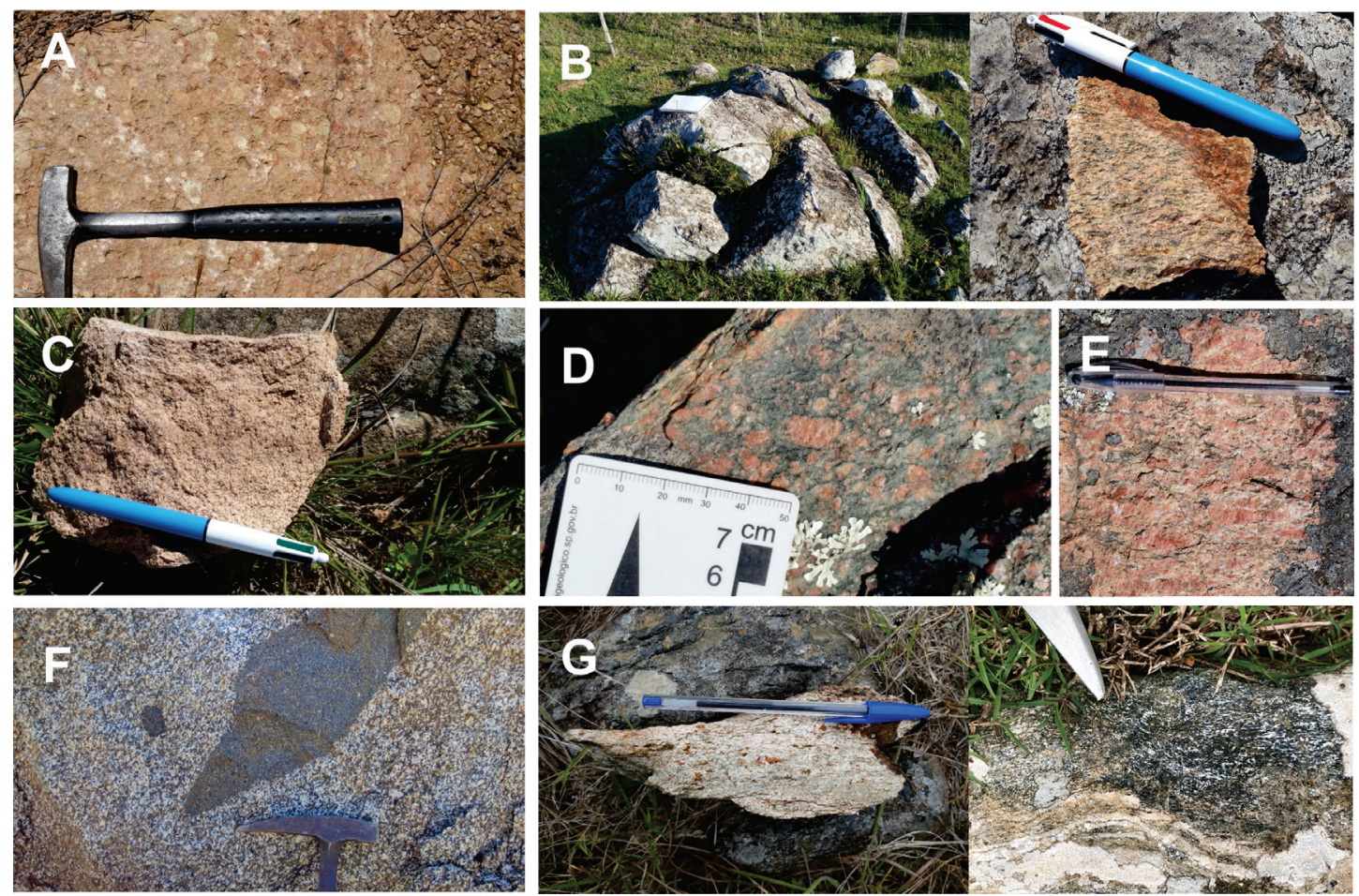

FIG. 3. A. General view of Illescas rapakivi granite (sample NP-02); B. General view of foliated granitoid (sample NP-07) at the Cerro de las Cuentas; C. Cerro Chato granite (sample NP-08); D. Mylonitic granite inside the Cueva del Tigre shear zone (NP-09A): recrystallized granite; E. Foliated granitoid (NP-09B); F. Deformed Zapicán diorite (NP-10); G. Foliated leucogranite of the La China Complex (NP-11) that shows irregular compositional bands, with amphibole and quartz-feldspathic rich bands. 
staurolite and garnet in the south (Hartmann et al., 2001). According to Oyhantçabal et al. (2018) and Loureiro et al. (2019), along its southeastern limit, represented by the Retamosa thrust (this work), the unit presents small flakes intercalated with supracrustal rocks of the Dom Feliciano Belt.

\subsection{Valentines Block}

The Valentines Block (Preciozzi et al., 1979) is located between the Sarandí del Yí shear zone and the Cueva del Tigre shear zone, next to the Pavas Block (Fig. 2). It is characterized by metatrondhjemites, metagranites and metatonalites. Ferriferous conglomerates, magnetite-rich quartzites, banded magneto-augite quartzites, BIFs, also cherts have been described, as well as amphibolites, pyroxenites, garnet-pyroxene quartzites and forsteritediopside marbles (e.g., Bossi and Umpierre, 1969; Masquelin, 2006; Oyhantçabal et al., 2011; Oriolo et al., 2016a). The Valentines Block is constituted by the Valentines-Rivera Granulitic Complex (Oyhantçabal et al., 2011), and a few Proterozoic igneous intrusions. Lithologically, the ValentinesRivera Granulitic Complex is composed mainly by granulitic gneisses, para-gneisses, meta-pyroxenites, magnetite-augite quartzites, meta-tonalites, metatrondjemites, migmatites, among others (Mallmann et al., 2007; Oyhantçabal et al., 2011 and references therein). The supracrustal rocks (paragneisses, quartzites, BIF) of this complex are included in the Valentines Formation (Bossi et al., 1965). The granulites are correlated and grouped with similar rocks from Brazil in the Taquarembó Block (Hartmann et al., 1999; Oyhantçabal et al., 2011; and references therein) and with the Santa María Chico Complex in Rio Grande do Sul (Massonne et al., 2001; and references therein).

Within this block, Campal and Schipilov (1995) described the occurrence of a large A-type rapakivi granite (Illescas Batholith, Fig. 3). It has a coarse- to medium-grain texture, with K-feldspar, plagioclase, quartz, amphibole, and biotite. It presents ductile deformation in its borders and it is cut by Sarandí del Yí shear zone (Campal and Schipilov, 1995). Gaucher and Blanco (2014) based on few and partial geochemical data suggested a within plate tectonic environment. On the contrary, Oriolo et al. (2019) conducted a geochemical study concluding that the Illescas granite exhibits meta- to peraluminous nature with dominant shoshonitic and ferroan composition, suggesting a post-collisional/post-orogenic setting.

\subsection{Rivera Block}

The Rivera Block (Preciozzi et al., 1979) is in the northeastern portion of Uruguay (Figs. 1 and 4) and is an isolated block surrounded by Palaeozoic sedimentary rocks from the Paraná Basin. The basement is a bimodal felsic-mafic association that underwent high-grade metamorphism constituted by granulitic othogneisses and subordinate orthopyroxene and garnet-bearing mafic granulites, grouped by Oyhantçabal et al. (2012) in the Valentines-Rivera Granulitic Complex. The regional structural trend is EW, affected by ductile shear zones (Fig. 4), attributed originally to the Brasiliano orogeny by Cordani and Soliani (1990).

In addition, the rocks of the Vichadero Formation are interleyed with the Valentines-Rivera Granulitic Complex (Oyhantçabal et al., 2012). This formation is composed of pyroxene fels, sillimanite gneisses, quartzites, forsterite marbles, metabasites, BIFs, calc-silicate rocks, and manganese-formations (Ellis De Luca, 1998). Important ore deposits (gold) occur related to the regional structural trend.

\subsection{Neoproterozoic cover rocks, granitic plutons, and shear zones}

In the Valentines Block, the Neoproterozoic cover is represented by the Cerro San Francisco and Cerros Victoria Formations, made up by metasandstones and meta-subarkoses, and by the oolitic and stromatolitic meta-limestone with low metamorphic grade, respectively, deposited in restricted sedimentary basin (Montaña and Sprechmann, 1993). In the Rivera Block, some outcrops of low-grade metamorphic rocks are described (e.g., Preciozzi et al., 1985; Gaucher, 2000).

An important Neoproterozoic magmatism, related to the Brasiliano orogenic cycle, crops out all over the Nico Pérez Terrane (Preciozzi et al., 1979; Cordani and Soliani, 1990; Oyhtançabal et al., 2012; Oriolo et al., 2016a; among others). Foliated granitic plutons occupy about $30 \%$ of the area covered by the Nico Pérez Terrane (Fig. 2). The granitoids are syn-, late-, to post-orogenic and they are coupled with the regional structures with NE-SW trends related to the Brasiliano Orogeny. Two of them, the Cerro 


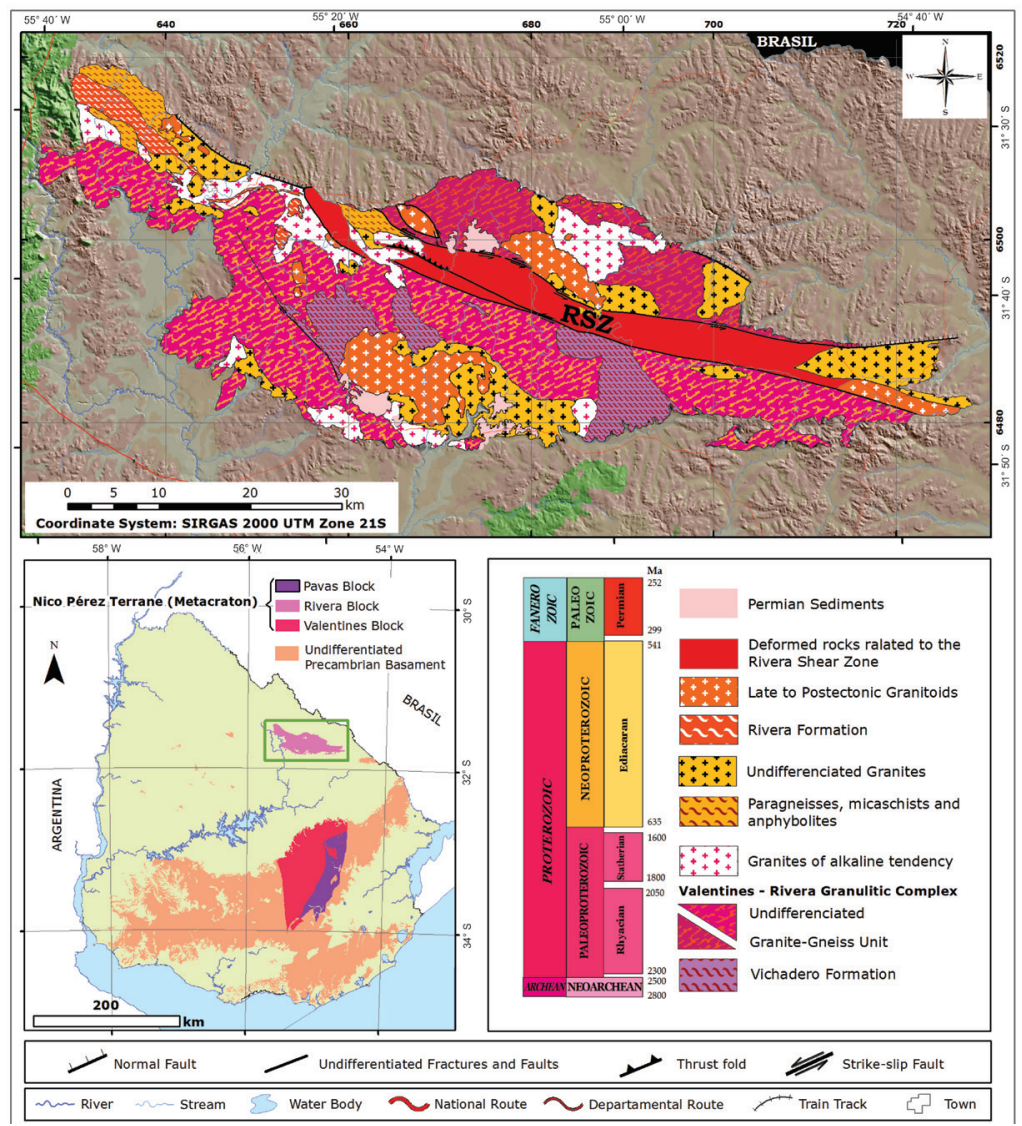

FIG. 4. Rivera Block geological map, modified from Loureiro (2008).

de las Cuentas and the Cerro Chato granites (Fig. 3), are studied in this work (see Section 4).

The most important shear zone that affects the Nico Pérez Terrane is the Paleoproterozoic Sarandí del Yí shear zone (Fig. 1). It comprises up to $13 \mathrm{~km}$ wide and more than $250 \mathrm{~km}$ long in the north-south direction (Gómez Rifas, 1989). Preciozzi et al. (1979) were the first to recognize this feature, with a $\mathrm{N} 10^{\circ}$ structural trend developed between the Sarandí of Yí town (Durazno department) and the Sierra de Las Ánimas Complex (Fig. 1). Bossi and Campal (1992) described it as a dextral megashear zone, based on features like the Florida dike swarm flexure, which are clearly well defined in the aerogeophysical survey (Dinamige, 2016). Oyhantçabal et al. (1993) suggested that this shear zone was reactivated with a sinistral sense during the Brasiliano orogenic cycle. Furthermore, Oriolo et al. (2016b) proposed this shear zone is due to the collision of the Nico Pérez Terrane and the Río de La Plata Craton, suggesting the onset of the deformation at 630-625 Ma, with dextral shearing up to $596 \mathrm{Ma}$ and subsequent sinistral shearing at 594-584 Ma.

Another important shear zone occurring in the Nico Pérez Terrane is the Cueva del Tigre shear zone (Preciozzi et al., 1979) developed at the boundary between the Valentines and Pavas blocks (Fig. 2). It is represented by a group of granitic mylonites, schists and phyllonites. This shear zone affected both, the Archean basement and the Neoproterozoic intrusions (Oriolo et al., 2016b).

\section{Previous radiometric data of the Nico Pérez Terrane}

The available ages for the three structural blocks of Nico Pérez Terrane are displayed in the table 1. The pioneer works of Soliani (1986) and Cordani and Soliani (1990) reported Paleoproterozoic and Neoproterozoic ages for the Rivera Block. These 
TABLE 1. GEOCHRONOLOGICAL AVAILABLE DATA FOR THE NICO PÉREZ TERRANE.

\begin{tabular}{|c|c|c|c|c|c|c|}
\hline Unit & Age (Ma) & Method & Litology & Interpretation & $\begin{array}{c}\text { Event } \\
\text { Interpretation }\end{array}$ & Reference \\
\hline La China Complex & $2787 \pm 6$ & $\begin{array}{l}\mathrm{Zr}: \mathrm{LA}-\mathrm{ICP}-\mathrm{MS}: \\
\mathrm{U}-\mathrm{Pb}\end{array}$ & Deformed Granite & crystallization & $\begin{array}{l}\text { Neoarchean } \\
\text { Magmatism. } \\
\text { Event } 2 .\end{array}$ & Gaucher et al. (2014a) \\
\hline La China Complex & $3029 \pm 54$ & $\begin{array}{l}\mathrm{Zr}: \mathrm{LA}-\mathrm{ICP}-\mathrm{MS}: \\
\mathrm{U}-\mathrm{Pb}\end{array}$ & $\begin{array}{l}\text { Biotitic } \\
\text { Orthogneiss }\end{array}$ & crystallization & $\begin{array}{l}\text { Mesoarchean } \\
\text { Magmatism. } \\
\text { Event } 1 .\end{array}$ & Gaucher et al. (2010a) \\
\hline La China Complex & $3096 \pm 45$ & $\begin{array}{l}\mathrm{Zr}: \mathrm{LA}-\mathrm{ICP}-\mathrm{MS}: \\
\mathrm{U}-\mathrm{Pb}\end{array}$ & Metatonalite & crystallization & $\begin{array}{l}\text { Mesoarchean } \\
\text { Magmatism. } \\
\text { Event } 1 .\end{array}$ & Gaucher et al. (2011) \\
\hline La China Complex & $2690 \pm 42$ & $\begin{array}{l}\mathrm{Zr}: \mathrm{LA}-\mathrm{ICP}-\mathrm{MS}: \\
\mathrm{U}-\mathrm{Pb}\end{array}$ & Metatonalite & metamorphism & $\begin{array}{l}\text { Neoarchean } \\
\text { Metamorfism. } \\
\text { Event } 2 .\end{array}$ & Gaucher et al. (2011) \\
\hline La China Complex & $2718 \pm 8$ & $\begin{array}{l}\mathrm{Zr}: \mathrm{LA}-\mathrm{ICP}-\mathrm{MS}: \\
\mathrm{U}-\mathrm{Pb}\end{array}$ & $\begin{array}{l}\text { Amphybolic } \\
\text { Orthogneiss }\end{array}$ & crystallization & $\begin{array}{l}\text { Neoarchean } \\
\text { Magmatism. } \\
\text { Event } 2 .\end{array}$ & Gaucher et al. (2014a) \\
\hline La China Complex & $3408 \pm 16$ & Zr-SHRIMP U-Pb & Metatonalite & inheritance & $\begin{array}{l}\text { Palaeoaorchean } \\
\text { basement } \\
\text { inheritance }\end{array}$ & Hartmann et al. (2001) \\
\hline La China Complex & $3100 \pm 100$ & Zr-SHRIMP U-Pb & Metatonalite & crystallization & $\begin{array}{l}\text { Mesoarchean } \\
\text { Magmatism. } \\
\text { Event } 1 .\end{array}$ & Hartmann et al. (2001) \\
\hline La China Complex & $2721 \pm 7$ & Zr-SHRIMP U-Pb & Metatonalite & metamorphism & $\begin{array}{l}\text { Neoarchean } \\
\text { Metamorfism. } \\
\text { Event } 2 .\end{array}$ & Hartmann et al. (2001) \\
\hline La China Complex & $2707 \pm 6.9$ & Zr-SHRIMP U-Pb & $\begin{array}{l}\text { Foliated } \\
\text { Leucogranite }\end{array}$ & crystallization & $\begin{array}{l}\text { Neoarchean } \\
\text { Magmatism. } \\
\text { Event } 2 .\end{array}$ & This work \\
\hline Las Tetas Complex & $3222 \pm 5$ & Zr-SHRIMP U-Pb & $\begin{array}{l}\text { Muscovitic } \\
\text { Quartzite }\end{array}$ & source age & $\begin{array}{l}\text { Palaeo to } \\
\text { Mesorchean } \\
\text { Source age. }\end{array}$ & Hartmann et al. (2001) \\
\hline Las Tetas Complex & $3263 \pm 14$ & Zr-SHRIMP U-Pb & $\begin{array}{l}\text { Muscovitic } \\
\text { Quartzite }\end{array}$ & source age & $\begin{array}{l}\text { Palaeo to } \\
\text { Mesorchean } \\
\text { Source age. }\end{array}$ & Hartmann et al. (2001) \\
\hline Las Tetas Complex & $3181 \pm 6$ & Zr-SHRIMP U-Pb & $\begin{array}{l}\text { Muscovitic } \\
\text { Quartzite }\end{array}$ & source age & $\begin{array}{l}\text { Palaeo to } \\
\text { Mesorchean } \\
\text { Source age. }\end{array}$ & Hartmann et al. (2001) \\
\hline Las Tetas Complex & $3146 \pm 4$ & Zr-SHRIMP U-Pb & $\begin{array}{l}\text { Muscovitic } \\
\text { Quartzite }\end{array}$ & source age & $\begin{array}{l}\text { Palaeo to } \\
\text { Mesorchean } \\
\text { Source age. }\end{array}$ & Hartmann et al. (2001) \\
\hline $\begin{array}{l}\text { Valentines } \\
\text { Formation }\end{array}$ & $2968 \pm 12$ & Zr-SHRIMP U-Pb & Metaconglomerate & source age & $\begin{array}{l}\text { Palaeo to } \\
\text { Mesorchean } \\
\text { Source age. } \\
\text { Event } 1 .\end{array}$ & Hartmann et al. (2001) \\
\hline $\begin{array}{l}\text { Valentines } \\
\text { Formation }\end{array}$ & $2762 \pm 8$ & Zr-SHRIMP U-Pb & Metaconglomerate & source age & $\begin{array}{l}\text { Neoarchean } \\
\text { Source age. } \\
\text { Event } 2 .\end{array}$ & Hartmann et al. (2001) \\
\hline VRGC & $2619 \pm 8$ & Zr-SHRIMP U-Pb & Granulite & inheritance & $\begin{array}{l}\text { Neoarchean } \\
\text { basement } \\
\text { inheritance }\end{array}$ & Santos et al. (2003) \\
\hline VRGC & $2535 \pm 12$ & Zr-SHRIMP U-Pb & Granulite & inheritance & $\begin{array}{l}\text { Neoarchean } \\
\text { basement } \\
\text { inheritance }\end{array}$ & Santos et al. (2003) \\
\hline VRGC & $2224 \pm 4$ & Zr-SHRIMP U-Pb & Granulite & inheritance & $\begin{array}{l}\text { Transamazonic } \\
\text { basement } \\
\text { inheritance }\end{array}$ & Santos et al. (2003) \\
\hline
\end{tabular}


table 1 continued.

\begin{tabular}{|c|c|c|c|c|c|c|}
\hline Unit & Age (Ma) & Method & Litology & Interpretation & $\begin{array}{c}\text { Event } \\
\text { Interpretation }\end{array}$ & Reference \\
\hline VRGC & $2163 \pm 8$ & Zr-SHRIMP U-Pb & Granulite & crystallization & $\begin{array}{l}\text { Transamazonic } \\
\text { Magmatism }\end{array}$ & Santos et al. (2003) \\
\hline VRGC & $2058 \pm 3$ & Zr-SHRIMP U-Pb & Granulite & metamorphism & $\begin{array}{l}\text { Transamazonic } \\
\text { Metamorfism }\end{array}$ & Santos et al. (2003) \\
\hline VRGC & $2106 \pm 21$ & $\begin{array}{l}\text { Zr: LA-ICP-MS: } \\
\text { U-Pb }\end{array}$ & $\begin{array}{l}\text { Felsic } \\
\text { Orthogneiss }\end{array}$ & crystallization & $\begin{array}{l}\text { Transamazonic } \\
\text { Magmatism }\end{array}$ & Oriolo et al. (2016a) \\
\hline VRGC & ca. 2700 & $\begin{array}{l}\text { Zr: LA-ICP-MS: } \\
\text { U-Pb }\end{array}$ & $\begin{array}{l}\text { Felsic } \\
\text { Orthogneiss }\end{array}$ & inheritance & $\begin{array}{l}\text { Neoarchean } \\
\text { basement } \\
\text { inheritance }\end{array}$ & Oriolo et al. (2016a) \\
\hline VRGC & $2048 \pm 11$ & $\mathrm{Zr}:$ SHRIMP U-Pb & $\begin{array}{l}\text { Mylonitized } \\
\text { Granite }\end{array}$ & crystallization & $\begin{array}{l}\text { Transamazonic } \\
\text { Magmatism }\end{array}$ & Oriolo et al. (2016b) \\
\hline VRGC & $2163 \pm 8$ & Zr-SHRIMP U-Pb & Trondhjemite & inheritance & $\begin{array}{l}\text { Transamazonic } \\
\text { basement } \\
\text { inheritance }\end{array}$ & Santos et al. (2003) \\
\hline VRGC & $2140 \pm 6$ & Zr-SHRIMP U-Pb & Trondhjemite & crystallization & $\begin{array}{l}\text { Transamazonic } \\
\text { Magmatism }\end{array}$ & Santos et al. (2003) \\
\hline VRGC & $2077 \pm 6$ & Zr-SHRIMP U-Pb & Trondhjemite & metamorphism & $\begin{array}{l}\text { Transamazonic } \\
\text { Metamorfism }\end{array}$ & Santos et al. (2003) \\
\hline VRGC & $2171.7 \pm 8.4$ & Zr-SHRIMP U-Pb & Leucocratic Gneiss & crystallization & $\begin{array}{l}\text { Transamazonic } \\
\text { Magmatism }\end{array}$ & Oyhantçabal et al. (2012) \\
\hline VRGC & $2139 \pm 73$ & Zr-SHRIMP U-Pb & Leucocratic Gneiss & metamorphism & $\begin{array}{l}\text { Transamazonic } \\
\text { Metamorfism }\end{array}$ & Oyhantçabal et al. (2012) \\
\hline VRGC & $587 \pm 19$ & Zr-SHRIMP U-Pb & Leucocratic Gneiss & metamorphism & $\begin{array}{l}\text { Transamazonic } \\
\text { Metamorfism }\end{array}$ & Oyhantçabal et al. (2012) \\
\hline VRGC & $1976 \pm 3$ & Mn-Th-U-Pb-EMP & Leucocratic Gneiss & metamorphism & $\begin{array}{l}\text { Transamazonic } \\
\text { Metamorfism }\end{array}$ & Oyhantçabal et al. (2012) \\
\hline VRGC & $2147 \pm 8.7$ & Zr-SHRIMP U-Pb & Mesocratic Gneiss & crystallization & $\begin{array}{l}\text { Transamazonic } \\
\text { Magmatism }\end{array}$ & Oyhantçabal et al. (2012) \\
\hline VRGC & $2113.7 \pm 3.1$ & Zr-SHRIMP U-Pb & Mesocratic Gneiss & crystallization & $\begin{array}{l}\text { Transamazonic } \\
\text { Magmatism }\end{array}$ & Oyhantçabal et al. (2012) \\
\hline VRGC & $1981 \pm 2$ & Mn-Th-U-Pb-EMP & Mesocratic Gneiss & metamorphism & $\begin{array}{l}\text { Transamazonic } \\
\text { Metamorfism }\end{array}$ & Oyhantçabal et al. (2012) \\
\hline VRGC & $2094 \pm 17$ & Zr-SHRIMP U-Pb & Mesocratic Gneiss & metamorphism & $\begin{array}{l}\text { Transamazonic } \\
\text { Metamorfism }\end{array}$ & Oyhantçabal et al. (2012) \\
\hline VRGC & $2095 \pm 15$ & $\begin{array}{l}\mathrm{Zr}: \text { LA-ICP-MS: } \\
\mathrm{U}-\mathrm{Pb}\end{array}$ & Mafic Granulite & crystallization & $\begin{array}{l}\text { Transamazonic } \\
\text { Magmatism }\end{array}$ & Oriolo et al. (2016a) \\
\hline VRGC & $2041 \pm 24$ & $\begin{array}{l}\text { Zr: LA-ICP-MS: } \\
\text { U-Pb }\end{array}$ & Mafic Granulite & metamorphism & $\begin{array}{l}\text { Transamazonic } \\
\text { Metamorfism }\end{array}$ & Oriolo et al. (2016a) \\
\hline VRGC & $2069 \pm 16$ & $\begin{array}{l}\mathrm{Zr}: \text { LA-ICP-MS: } \\
\mathrm{U}-\mathrm{Pb}\end{array}$ & Felsic Orthogneiss & metamorphism & $\begin{array}{l}\text { Transamazonic } \\
\text { Metamorfism }\end{array}$ & Oriolo et al. (2016a) \\
\hline VRGC & $2087 \pm 7.3$ & $\begin{array}{l}\text { Zr: LA-ICP-MS: } \\
\text { U-Pb }\end{array}$ & TG Gneiss & crystallization & $\begin{array}{l}\text { Transamazonic } \\
\text { Magmatism }\end{array}$ & Oriolo et al. (2016a) \\
\hline VRGC & $1857 \pm 45$ & $\begin{array}{l}\text { Zr: LA-ICP-MS: } \\
\text { U-Pb }\end{array}$ & TG Gneiss & metamorphism & $?$ & Oriolo et al. (2016a) \\
\hline VRGC & $1790 \pm 50$ & $\mathrm{Rb} / \mathrm{Sr} \mathrm{WR}$ & Gneiss & $?$ & $?$ & Cordani and Soliani (1990) \\
\hline VRGC & $2140 \pm 150$ & $\mathrm{Rb} / \mathrm{Sr} \mathrm{WR}$ & Gneiss & metamorphism & $\begin{array}{l}\text { Transamazonic } \\
\text { metamorphism }\end{array}$ & Cordani and Soliani (1990) \\
\hline VRGC & $2030 \pm 70$ & $\mathrm{Rb} / \mathrm{Sr} \mathrm{WR}$ & Gneiss & metamorphism & $\begin{array}{l}\text { Transamazonic } \\
\text { metamorphism }\end{array}$ & Cordani and Soliani (1990) \\
\hline
\end{tabular}


table 1 continued.

\begin{tabular}{|c|c|c|c|c|c|c|}
\hline Unit & Age (Ma) & Method & Litology & Interpretation & $\begin{array}{c}\text { Event } \\
\text { Interpretation }\end{array}$ & Reference \\
\hline VRGC & $2190 \pm 80$ & $\mathrm{Rb} / \mathrm{Sr} \mathrm{WR}$ & Gneiss & metamorphism & $\begin{array}{l}\text { Transamazonic } \\
\text { metamorphism }\end{array}$ & Cordani and Soliani (1990) \\
\hline VRGC & $2250 \pm 10$ & $\mathrm{Rb} / \mathrm{Sr} \mathrm{WR}$ & Gneiss & metamorphism & $\begin{array}{l}\text { Transamazonic } \\
\text { metamorphism }\end{array}$ & Cordani and Soliani (1990) \\
\hline VRGC & $2180 \pm 70$ & $\mathrm{Rb} / \mathrm{Sr} \mathrm{WR}$ & Gneiss & metamorphism & $\begin{array}{l}\text { Transamazonic } \\
\text { metamorphism }\end{array}$ & Cordani and Soliani (1990) \\
\hline VRGC & $946 \pm 31$ & $\mathrm{~K}-\mathrm{Ar} \mathrm{Kf}$ & Gneiss & metamorphism & Tonian event? & Cordani and Soliani (1990) \\
\hline VRGC & $968 \pm 14$ & $\mathrm{~K}-\mathrm{Ar} \mathrm{Kf}$ & Gneiss & metamorphism & Tonian event? & Cordani and Soliani (1990) \\
\hline VRGC & $2200 \pm 60$ & $\mathrm{Rb} / \mathrm{Sr} \mathrm{WR}$ & Gneiss & metamorphism & $\begin{array}{l}\text { Transamazonic } \\
\text { metamorphism }\end{array}$ & Cordani and Soliani (1990) \\
\hline VRGC & $2220 \pm 90$ & $\mathrm{Rb} / \mathrm{Sr} \mathrm{WR}$ & Gneiss & metamorphism & $\begin{array}{l}\text { Transamazonic } \\
\text { metamorphism }\end{array}$ & Cordani and Soliani (1990) \\
\hline VRGC & $2240 \pm 110$ & $\mathrm{Rb} / \mathrm{Sr} \mathrm{WR}$ & Gneiss & metamorphism & $\begin{array}{l}\text { Transamazonic } \\
\text { metamorphism }\end{array}$ & Cordani and Soliani (1990) \\
\hline VRGC & $560 \pm 70$ & $\mathrm{Rb} / \mathrm{Sr} \mathrm{WR}$ & Metatonalite & magmatism & $\begin{array}{l}\text { Brasiliano } \\
\text { Magmatism }\end{array}$ & Cordani and Soliani (1990) \\
\hline VRGC & $540 \pm 90$ & $\mathrm{Rb} / \mathrm{Sr} \mathrm{WR}$ & Tonalite & magmatism & $\begin{array}{l}\text { Brasiliano } \\
\text { Magmatism }\end{array}$ & Cordani and Soliani (1990) \\
\hline $\begin{array}{l}\text { Undifferenciated } \\
\text { granites (RB) }\end{array}$ & $507 \pm 12$ & $\mathrm{~K}-\mathrm{Ar} \mathrm{Pg}$ & Tonalite & magmatism & $\begin{array}{l}\text { Brasiliano } \\
\text { Magmatism }\end{array}$ & Cordani and Soliani (1990) \\
\hline $\begin{array}{l}\text { Undifferenciated } \\
\text { granites (RB) }\end{array}$ & $586 \pm 22$ & $\mathrm{~K}-\mathrm{Ar} \mathrm{Hbl}$ & Tonalite & magmatism & $\begin{array}{l}\text { Brasiliano } \\
\text { Magmatism }\end{array}$ & Cordani and Soliani (1990) \\
\hline $\begin{array}{l}\text { Undifferenciated } \\
\text { granites }(\mathrm{RB})\end{array}$ & $536 \pm 11$ & K-Ar WR & Microgranite & magmatism & $\begin{array}{l}\text { Brasiliano } \\
\text { Magmatism }\end{array}$ & Cordani and Soliani (1990) \\
\hline $\begin{array}{l}\text { Undifferenciated } \\
\text { granites }(\mathrm{RB})\end{array}$ & $584 \pm 15$ & $\mathrm{~K}-\mathrm{Ar} \mathrm{Pg}$ & Granodiorite & magmatism & $\begin{array}{l}\text { Brasiliano } \\
\text { Magmatism }\end{array}$ & Cordani and Soliani (1990) \\
\hline $\begin{array}{l}\text { Undifferenciated } \\
\text { granites }(\mathrm{RB})\end{array}$ & $589 \pm 8$ & $\mathrm{~K}-\mathrm{Ar} \mathrm{Bt}$ & Granodiorite & magmatism & $\begin{array}{l}\text { Brasiliano } \\
\text { Magmatism }\end{array}$ & Cordani and Soliani (1990) \\
\hline $\begin{array}{l}\text { Undifferenciated } \\
\text { granites (RB) }\end{array}$ & $576 \pm 10$ & $\mathrm{~K}-\mathrm{Ar} \mathrm{Bt}$ & Granodiorite & magmatism & $\begin{array}{l}\text { Brasiliano } \\
\text { Magmatism }\end{array}$ & Cordani and Soliani (1990) \\
\hline $\begin{array}{l}\text { Undifferenciated } \\
\text { granites (RB) }\end{array}$ & $499 \pm 12$ & $\mathrm{~K}-\mathrm{Ar} \mathrm{Pg}$ & Granodiorite & magmatism & $\begin{array}{l}\text { Brasiliano } \\
\text { Magmatism }\end{array}$ & Cordani and Soliani (1990) \\
\hline $\begin{array}{l}\text { Undifferenciated } \\
\text { granites (RB) }\end{array}$ & $523 \pm 13$ & K-Ar WR & Microgranite & magmatism & $\begin{array}{l}\text { Brasiliano } \\
\text { Magmatism }\end{array}$ & Cordani and Soliani (1990) \\
\hline $\begin{array}{l}\text { Undifferenciated } \\
\text { granites (RB) }\end{array}$ & $540 \pm 90$ & $\mathrm{Rb} / \mathrm{Sr} \mathrm{WR}$ & Granite & magmatism & $\begin{array}{l}\text { Brasiliano } \\
\text { Magmatism }\end{array}$ & Cordani and Soliani (1990) \\
\hline $\begin{array}{l}\text { Undifferenciated } \\
\text { granites (RB) }\end{array}$ & $515 \pm 11$ & $\mathrm{~K}-\mathrm{Ar} \mathrm{Bt}$ & Granite & magmatism & $\begin{array}{l}\text { Brasiliano } \\
\text { Magmatism }\end{array}$ & Cordani and Soliani (1990) \\
\hline $\begin{array}{l}\text { Undifferenciated } \\
\text { granites (RB) }\end{array}$ & $537 \pm 15$ & K-Ar WR & Microgranite & magmatism & $\begin{array}{l}\text { Brasiliano } \\
\text { Magmatism }\end{array}$ & Cordani and Soliani (1990) \\
\hline $\begin{array}{l}\text { Sobresaliente } \\
\text { Granite }\end{array}$ & $2043 \pm 44$ & Zr-SHRIMP U-Pb & Quartz Monzonite & crystallization & $\begin{array}{l}\text { Brasiliano } \\
\text { Magmatism }\end{array}$ & Oyhantçabal et al. (2012) \\
\hline $\begin{array}{l}\text { Sobresaliente } \\
\text { Granite }\end{array}$ & $578.1 \pm 5.8$ & Zr-SHRIMP U-Pb & Quartz Monzonite & crystallization & $\begin{array}{l}\text { Brasiliano } \\
\text { Magmatism }\end{array}$ & Oyhantçabal et al. (2012) \\
\hline Las Flores Granite & $586 \pm 2.7$ & Zr-SHRIMP U-Pb & Granite & crystallization & $\begin{array}{l}\text { Brasiliano } \\
\text { Magmatism }\end{array}$ & Oyhantçabal et al. (2012) \\
\hline Las Flores Granite & $578 \pm 10$ & $\mathrm{~K}-\mathrm{Ar} \mathrm{Bt}$ & Granite & crystallization & $\begin{array}{l}\text { Brasiliano } \\
\text { Magmatism }\end{array}$ & Oyhantçabal et al. (2012) \\
\hline Las Flores Granite & $579 \pm 20$ & $\mathrm{~K}-\mathrm{Ar} \mathrm{Bt}$ & Granodiorite & magmatism & $\begin{array}{l}\text { Brasiliano } \\
\text { Magmatism }\end{array}$ & Cordani and Soliani (1990) \\
\hline
\end{tabular}


table 1 continued.

\begin{tabular}{|c|c|c|c|c|c|c|}
\hline Unit & Age (Ma) & Method & Litology & Interpretation & $\begin{array}{c}\text { Event } \\
\text { Interpretation }\end{array}$ & Reference \\
\hline Las Flores Granite & $370 \pm 7$ & $\mathrm{~K}-\mathrm{Ar} \mathrm{Bt}$ & Granodiorite & magmatism & $\begin{array}{l}\text { Brasiliano } \\
\text { Magmatism }\end{array}$ & Cordani and Soliani (1990) \\
\hline Las Flores Granite & $1200 \pm ?(\mathrm{sic})$ & $\mathrm{Rb} / \mathrm{Sr} \mathrm{WR}$ & Granodiorite & magmatism & $\begin{array}{l}\text { Brasiliano } \\
\text { Magmatism }\end{array}$ & Cordani and Soliani (1990) \\
\hline Amarillo granite & $2530 \pm 26$ & $\begin{array}{l}\text { Zr: LA-ICP-MS: } \\
\mathrm{U}-\mathrm{Pb}\end{array}$ & Leucogranite & inheritance & $\begin{array}{l}\text { Archean } \\
\text { inheritance }\end{array}$ & Oriolo et al. (2016a) \\
\hline Amarillo Granite & $596 \pm 2.3$ & $\begin{array}{l}\mathrm{Zr}: \mathrm{LA}-\mathrm{ICP}-\mathrm{MS}: \\
\mathrm{U}-\mathrm{Pb}\end{array}$ & Leucogranite & crystallization & $\begin{array}{l}\text { Brasiliano } \\
\text { Magmatism }\end{array}$ & Oriolo et al. (2016a) \\
\hline Illescas Granite & $1760 \pm 30$ & $\mathrm{Rb} / \mathrm{Sr}$ & Rapakivi Granite & crystallization & $\begin{array}{l}\text { Statherian } \\
\text { Extensional } \\
\text { Magmatism }\end{array}$ & Bossi and Campal (1992) \\
\hline Illescas Granite & $1784 \pm 5$ & $\mathrm{~Pb}-\mathrm{Pb}$ & Rapakivi Granite & crystallization & $\begin{array}{l}\text { Statherian } \\
\text { Extensional } \\
\text { Magmatism }\end{array}$ & $\begin{array}{l}\text { Campal and Schipilov } \\
\text { (1995) }\end{array}$ \\
\hline Illescas Granite & $1734 \pm 11$ & Zr-SHRIMP U-Pb & Rapakivi Granite & crystallization & $\begin{array}{l}\text { Statherian } \\
\text { Extensional } \\
\text { Magmatism }\end{array}$ & This work \\
\hline Illescas Granite & $1768 \pm 11$ & U-Pb LA-ICP-MS & Rapakivi Granite & crystallization & $\begin{array}{l}\text { Statherian } \\
\text { Extensional } \\
\text { Magmatism }\end{array}$ & Oriolo et al. (2019) \\
\hline Zapican Diorite & $610.4 \pm 2.5$ & $\begin{array}{l}\mathrm{Zr}: \mathrm{LA}-\mathrm{ICP}-\mathrm{MS}: \\
\mathrm{U}-\mathrm{Pb}\end{array}$ & $\begin{array}{l}\text { Tonalite- } \\
\text { Granodiorite }\end{array}$ & crystallization & $\begin{array}{l}\text { Brasiliano } \\
\text { Magmatism }\end{array}$ & Oriolo et al. (2016a) \\
\hline Zapican Diorite & $3045 \pm 26$ & $\begin{array}{l}\text { Zr: LA-ICP-MS: } \\
\text { U-Pb }\end{array}$ & $\begin{array}{l}\text { Tonalite- } \\
\text { Granodiorite }\end{array}$ & inheritance & $\begin{array}{l}\text { Archean } \\
\text { inheritance }\end{array}$ & Oriolo et al. (2016a) \\
\hline Zapican Diorite & $585.8 \pm 5.7$ & Zr-SHRIMP U-Pb & Tonalitic Gneiss & crystallization & $\begin{array}{l}\text { Brasiliano } \\
\text { Magmatism }\end{array}$ & This work \\
\hline $\begin{array}{l}\text { Cerro de las } \\
\text { Cuentas Granite }\end{array}$ & $2118 \pm 62$ & Zr-SHRIMP U-Pb & Deformed Granite & inheritance & $\begin{array}{l}\text { Transamazonic } \\
\text { basement } \\
\text { inheritance }\end{array}$ & This work \\
\hline $\begin{array}{l}\text { Cerro de las } \\
\text { Cuentas Granite }\end{array}$ & $584.4 \pm 8.2$ & Zr-SHRIMP U-Pb & Deformed Granite & crystallization & $\begin{array}{l}\text { Brasiliano } \\
\text { Magmatism }\end{array}$ & This work \\
\hline $\begin{array}{l}\text { Cerro Chato } \\
\text { Granite }\end{array}$ & $\sim 2190$ & Zr-SHRIMP U-Pb & Granite & inheritance & $\begin{array}{l}\text { Transamazonic } \\
\text { basement } \\
\text { inheritance }\end{array}$ & This work \\
\hline $\begin{array}{l}\text { Cerro Chato } \\
\text { Granite }\end{array}$ & $\sim 1492$ & Zr-SHRIMP U-Pb & Granite & inheritance & $\begin{array}{l}\text { Mesoproterozoic } \\
\text { basement } \\
\text { inheritance }\end{array}$ & This work \\
\hline $\begin{array}{l}\text { Cerro Chato } \\
\text { Granite }\end{array}$ & $639 \pm 98$ & Zr-SHRIMP U-Pb & Granite & crystallization & $\begin{array}{l}\text { Brasiliano } \\
\text { Magmatism }\end{array}$ & This work \\
\hline $\begin{array}{l}\text { Cueva del Tigre } \\
\text { shear zone }\end{array}$ & $3087 \pm 13$ & Zr-SHRIMP U-Pb & $\begin{array}{l}\text { Mylonitized } \\
\text { Granite }\end{array}$ & inheritance & $\begin{array}{l}\text { Mesoarchean } \\
\text { basement } \\
\text { inheritance }\end{array}$ & This work \\
\hline $\begin{array}{l}\text { Cueva del Tigre } \\
\text { shear zone }\end{array}$ & $588.0 \pm 6.1$ & Zr-SHRIMP U-Pb & $\begin{array}{l}\text { Mylonitized } \\
\text { Granite }\end{array}$ & crystallization & $\begin{array}{l}\text { Brasiliano } \\
\text { Magmatism }\end{array}$ & This work \\
\hline $\begin{array}{l}\text { Cueva del Tigre } \\
\text { shear zone }\end{array}$ & $3089 \pm 11$ & Zr-SHRIMP U-Pb & $\begin{array}{l}\text { Mylonitized } \\
\text { Granite }\end{array}$ & crystallization & $\begin{array}{l}\text { Mesorchean } \\
\text { Magmatism. } \\
\text { Event } 1 .\end{array}$ & This work \\
\hline $\begin{array}{l}\text { Cueva del Tigre } \\
\text { shear zone }\end{array}$ & $3092 \pm 5$ & Zr-SHRIMP U-Pb & Granitic Mylonite & crystallization & $\begin{array}{l}\text { Mesorchean } \\
\text { Magmatism. } \\
\text { Event } 1 .\end{array}$ & This work \\
\hline $\begin{array}{l}\text { Cueva del Tigre } \\
\text { shear zone }\end{array}$ & $\sim 2142$ & Zr-SHRIMP U-Pb & Granitic Mylonite & metamorphism & $\begin{array}{l}\text { Transamazonic } \\
\text { Metamorfism }\end{array}$ & This work \\
\hline
\end{tabular}


table 1 continued.

\begin{tabular}{|c|c|c|c|c|c|c|}
\hline Unit & Age (Ma) & Method & Litology & Interpretation & $\begin{array}{c}\text { Event } \\
\text { Interpretation }\end{array}$ & Reference \\
\hline Rivera shear zone & $1935 \pm 9$ & Mn-Th-U-Pb-EMP & Mylonite & $?$ & - & Oyhantçabal et al. (2012) \\
\hline Rivera shear zone & $606 \pm 10$ & Mn-Th-U-Pb-EMP & Mylonite & shear & - & Oyhantçabal et al. (2012) \\
\hline Rivera shear zone & $510 \pm 18$ & Mn-Th-U-Pb-EMP & Mylonite & shear & - & Oyhantçabal et al. (2012) \\
\hline Rivera shear zone & $606 \pm 10.1$ & $\mathrm{~K}-\mathrm{Ar} \mathrm{Ms}$ & Mylonite & shear & - & Oyhantçabal et al. (2012) \\
\hline Rivera shear zone & $366 \pm 31$ & $\mathrm{~K}-\mathrm{Ar} \mathrm{Bt}$ & Cataclastic Granite & deformation & Ar lost & Cordani and Soliani (1990) \\
\hline Rivera shear zone & $414 \pm 29$ & $\mathrm{~K}-\mathrm{Ar} \mathrm{Bt}$ & Cataclastic Granite & deformation & Ar lost & Cordani and Soliani (1990) \\
\hline Rivera shear zone & $569 \pm 4$ & $\mathrm{Rb} / \mathrm{Sr} \mathrm{WR}$ & Granite & magmatism & $\begin{array}{l}\text { Brasiliano } \\
\text { Magmatism }\end{array}$ & Cordani and Soliani (1990) \\
\hline Rivera shear zone & $569 \pm 4$ & $\mathrm{Rb} / \mathrm{Sr} \mathrm{WR}$ & Microgranite & magmatism & $\begin{array}{l}\text { Brasiliano } \\
\text { Magmatism }\end{array}$ & Cordani and Soliani (1990) \\
\hline Rivera shear zone & $569 \pm 4$ & $\mathrm{Rb} / \mathrm{Sr} \mathrm{WR}$ & Granite & magmatism & $\begin{array}{l}\text { Brasiliano } \\
\text { Magmatism }\end{array}$ & Cordani and Soliani (1990) \\
\hline $\begin{array}{l}\text { Sarandí del Yi } \\
\text { shear zone }\end{array}$ & $2025 \pm 37$ & $\begin{array}{l}\mathrm{Zr}: \mathrm{LA}-\mathrm{ICP}-\mathrm{MS}: \\
\mathrm{U}-\mathrm{Pb}\end{array}$ & Mylonite & crystallization & $\begin{array}{l}\text { Transamazonic } \\
\text { Magmatism }\end{array}$ & Oriolo et al. (2016b) \\
\hline $\begin{array}{l}\text { Sarandí del Yi } \\
\text { shear zone }\end{array}$ & $623 \pm 5$ & $\begin{array}{l}\mathrm{Zr}: \mathrm{LA}-\mathrm{ICP}-\mathrm{MS}: \\
\mathrm{U}-\mathrm{Pb}\end{array}$ & Mylonite & $\begin{array}{l}\text { dynamo- } \\
\text { metamorphism }\end{array}$ & $\begin{array}{l}\text { Brasiliano } \\
\text { deformation }\end{array}$ & Oriolo et al. (2016b) \\
\hline $\begin{array}{l}\text { Sarandí del Yi } \\
\text { shear zone }\end{array}$ & $590.2 \pm 2.8$ & $\mathrm{Ar} / \mathrm{Ar} \mathrm{hbl}$ & Mylonite & $\begin{array}{l}\text { dynamo- } \\
\text { metamorphism }\end{array}$ & $\begin{array}{l}\text { Brasiliano } \\
\text { deformation }\end{array}$ & Oriolo et al. (2016b) \\
\hline $\begin{array}{l}\text { Sarandí del Yi } \\
\text { shear zone }\end{array}$ & $2048 \pm 11$ & $\begin{array}{l}\mathrm{Zr}: \mathrm{LA}-\mathrm{ICP}-\mathrm{MS}: \\
\mathrm{U}-\mathrm{Pb}\end{array}$ & Granitic Mylonite & crystallization & $\begin{array}{l}\text { Transamazonic } \\
\text { Magmatism }\end{array}$ & Oriolo et al. (2016b) \\
\hline $\begin{array}{l}\text { Sarandí del Yi } \\
\text { shear zone }\end{array}$ & $2115 \pm 38$ & $\begin{array}{l}\mathrm{Zr}: \mathrm{LA}-\mathrm{ICP}-\mathrm{MS}: \\
\mathrm{U}-\mathrm{Pb}\end{array}$ & $\begin{array}{l}\text { Deformed } \\
\text { Granitoid }\end{array}$ & crystallization & $\begin{array}{l}\text { Transamazonic } \\
\text { Magmatism }\end{array}$ & Oriolo et al. (2016b) \\
\hline $\begin{array}{l}\text { Sarandí del Yi } \\
\text { shear zone }\end{array}$ & $589.1 \pm 1.6$ & $\begin{array}{l}\mathrm{Zr}: \mathrm{LA}-\mathrm{ICP}-\mathrm{MS}: \\
\mathrm{U}-\mathrm{Pb}\end{array}$ & $\begin{array}{l}\text { Deformed } \\
\text { Granitoid }\end{array}$ & $\begin{array}{l}\text { dynamo- } \\
\text { metamorphism }\end{array}$ & $\begin{array}{l}\text { Brasiliano } \\
\text { deformation }\end{array}$ & Oriolo et al. (2016b) \\
\hline $\begin{array}{l}\text { Sarandí del Yi } \\
\text { shear zone }\end{array}$ & $2068.9 \pm 4.2$ & $\begin{array}{l}\mathrm{Zr}: \mathrm{LA}-\mathrm{ICP}-\mathrm{MS}: \\
\mathrm{U}-\mathrm{Pb}\end{array}$ & Ultramylonite & $\begin{array}{l}\text { dynamo- } \\
\text { metamorphism }\end{array}$ & $\begin{array}{l}\text { Transamazonic } \\
\text { Deformation }\end{array}$ & Oriolo et al. (2016b) \\
\hline $\begin{array}{l}\text { Sarandí del Yi } \\
\text { shear zone }\end{array}$ & $596.0 \pm 3.3$ & $\begin{array}{l}\mathrm{Zr}: \mathrm{LA}-\mathrm{ICP}-\mathrm{MS}: \\
\mathrm{U}-\mathrm{Pb}\end{array}$ & Mylonite & crystallization & $\begin{array}{l}\text { Brasiliano } \\
\text { Magmatism }\end{array}$ & Oriolo et al. (2016b) \\
\hline $\begin{array}{l}\text { Sarandí del Yi } \\
\text { shear zone }\end{array}$ & $600.1 \pm 3.4$ & $\mathrm{Ar} / \mathrm{Ar} \mathrm{Ms}$ & Mylonite & $?$ & $?$ & Oriolo et al. (2016b) \\
\hline $\begin{array}{l}\text { Sarandí del Yi } \\
\text { shear zone }\end{array}$ & $566 \pm 2.9$ & $\mathrm{Rb} / \mathrm{Sr} \mathrm{WR}$ & Mylonite & $\begin{array}{l}\text { dynamo- } \\
\text { metamorphism }\end{array}$ & $\begin{array}{l}\text { Brasiliano } \\
\text { deformation }\end{array}$ & Oriolo et al. (2016b) \\
\hline $\begin{array}{l}\text { Sarandí del Yi } \\
\text { shear zone }\end{array}$ & $594.41 \pm 0.99$ & $\mathrm{Ar} / \mathrm{Ar} \mathrm{Ms}$ & Mylonite & $\begin{array}{l}\text { dynamo- } \\
\text { metamorphism }\end{array}$ & $\begin{array}{l}\text { Brasiliano } \\
\text { deformation }\end{array}$ & Oriolo et al. (2016b) \\
\hline
\end{tabular}

authors obtained a $\mathrm{Rb}-\mathrm{Sr}(\mathrm{WR})$ isochron of $2250 \pm 60 \mathrm{Ma}$ for the quartz-feldspathic gneissic basement; also, Cordani and Soliani (1990) obtained several K-Ar ages in biotite, plagioclase, and whole rock around 500600 Ma. Recently, Santos et al. (2003), Oyhantçabal et al. (2012) and Oriolo et al. (2016a) obtained several $\mathrm{U}-\mathrm{Pb}$ single crystal zircon ages within the interval 2200-2000 Ma, for the high grade gneisses, which were interpreted as crystallization ages of the magmatic protoliths, confirming the paleoproterozoic age of the block. Oriolo et al. (2016a) and Oyhantçabal et al. (2012) also reported a few ages around $580 \mathrm{Ma}$ for Ediacaran granitic plutons.

In Valentines Block only a few U-Pb zircon ages have been obtained. Hartmann et al. (2001) reported Archean detrital zircon ages between 3000 and 
$2700 \mathrm{Ma}$ in a metaconglomerate (Valentines BIF's). For metagranites of this unit Santos et al. (2003), obtained ages between 2619 and 2058 Ma. More recently, Oriolo et al. (2016b) obtained two Paleoproterozoic ages for Valentines-Rivera Granulitic Complex of 2016 and 2048 Ma.

The Pavas Block also has a few geochronological data available. As mentioned above, Hartmann et al. (2001) obtained U-Pb zircon SHRIMP ages of $3400 \mathrm{Ma}$ for zircon cores and $2700 \mathrm{Ma}$ for zircon rims in a tonalitic orthogneiss of the La China Complex. Gaucher et al. (2011, 2014b) obtained a zircon U-Pb LA-ICP-MS age of $3096 \mathrm{Ma}$ for the same lithology, and detrital zircon ages of 3000 , 2970, 2760 and $2600 \mathrm{Ma}$ in a metaconglomerate of the Las Tetas Complex.

All the magmatic crystallization ages (e.g., zircon nuclei with $\mathrm{Th} / \mathrm{U}$ ratios greater than 0.1 ) reported by the different authors (Hartmann et al., 2001; Gaucher et al., 2011, 2014a and references therein) for the metatonalites from La China Complex can be grouped into Mesoarchean (3.03 Ga) and Neoarchean (2.79$2.72 \mathrm{Ga}$ ). On the other hand, metamorphic events (e.g., zircon rims with $\mathrm{Th} / \mathrm{U}$ ratios less than 0.1 ) show Mesoarchean $(3.1 \mathrm{Ga})$ and Neoarchean $(2.69 \mathrm{Ga})$ ages. Detrital zircons found in the Las Tetas Complex suggest Archean source areas and a maximum Mesoarchean deposition age (Loureiro et al., 2019 and references therein).

The intrusive Illescas granite was dated by Bossi and Campal (1992) by whole-rock Rb-Sr method, yielding $1760 \pm 32 \mathrm{Ma}$; later Campal and Schipilov (1995) reported a $\mathrm{Pb}-\mathrm{Pb}$ age of $1784 \pm 5 \mathrm{Ma}$. More recently Oriolo et al. (2019) obtained a zircon U-Pb LA-ICP-MS age of $1768 \pm 11$ Ma for this granite.

Younger ages reported in the Nico Pérez Terrane are $739 \pm 20 \mathrm{Ma}(\mathrm{Rb}-\mathrm{Sr} \mathrm{WR})$ for the Cerro Chato granite (Bossi and Campal, 1987), and 610 Ma for the Zapicán diorite that intrudes La China Complex (Oriolo et al., 2016a).

\section{Results of U-Pb zircon geochronology}

New U-Pb SHRIMP zircon results on eight samples of granitoid rocks are presented in this work with the goal of constraining the tectonic evolution of the Nico Pérez Terrane. The analytical data are included in table 2 . The zircon crystals were extracted from the samples by conventional methods, mounted in epoxy mounts, together with a few fragments of the TEMORA-2 standard, and polished. Then, cathodoluminescence images were obtained to reveal their internal structure to target the best sites for the U-Pb isotopic analyses. All measurements were made at Centro de Pesquisas Geocronológicas (CPGeo) of the University of São Paulo (USP), Brazil.

The eight samples were taken from six outcrops and were analyzed using the SHRIMP-II instrument of the CPGeo. Details of the analytical procedures, including the calibration methods, were presented by Williams (1998) and the work at the São Paulo laboratory was described by Sato et al. (2014). $\mathrm{U}$ abundance and $\mathrm{U} / \mathrm{Pb}$ values of the studied zircon crystals were calibrated against the Z6266 (903 ppm) and the TEMORA-2 (416.78 Ma) standards, respectively. Individual ages were determined from five successive scans of the mass spectrum, and the ages reported in the text are with 95\% confidence limits. Correction for common $\mathrm{Pb}$ was made using the measured ${ }^{204} \mathrm{~Pb}$, and the typical error component for the ${ }^{206} \mathrm{~Pb} /{ }^{238} \mathrm{U}$ ratios is less than 2 percent. The data were reduced by using a SQUID software and the Concordia diagrams were prepared using Isoplot /Ex (Ludwig, 2009).

Zircons are typically prismatic to equant, where several present oscillatory zoning. Some crystals present homogeneous cores, either bright or dark. In many cases the crystals are fragmented. The cathodoluminescence images of representative analyzed zircons are shown in figure 5 . The concordia diagrams for each analyzed sample are shown in figures 6 to 13 .

\subsection{Sample NP-07-Cerro de las Cuentas foliated granitoid}

This sample was collected at Cerro de las Cuentas granite, in the northernmost part of the Valentines Block (Fig. 2). This granite intrudes the ValentinesRivera Granulitic Complex, and it corresponds to an equigranular fine-grained foliated granite, composed of quartz $+\mathrm{K}$-feldspar+plagioclase + biotite. The deformation is not observed in the outcrop scale, but undulose extinction and bulging recrystallization in quartz crystals occur. Zircon crystals are mostly prismatic showing oscillatory zoning and some recrystallization phenomena (Fig. 5b). A total of nine spots out of six zircon crystals were analyzed (Table 2). Three zircon grains (\#1, \#2 and \#7) were 


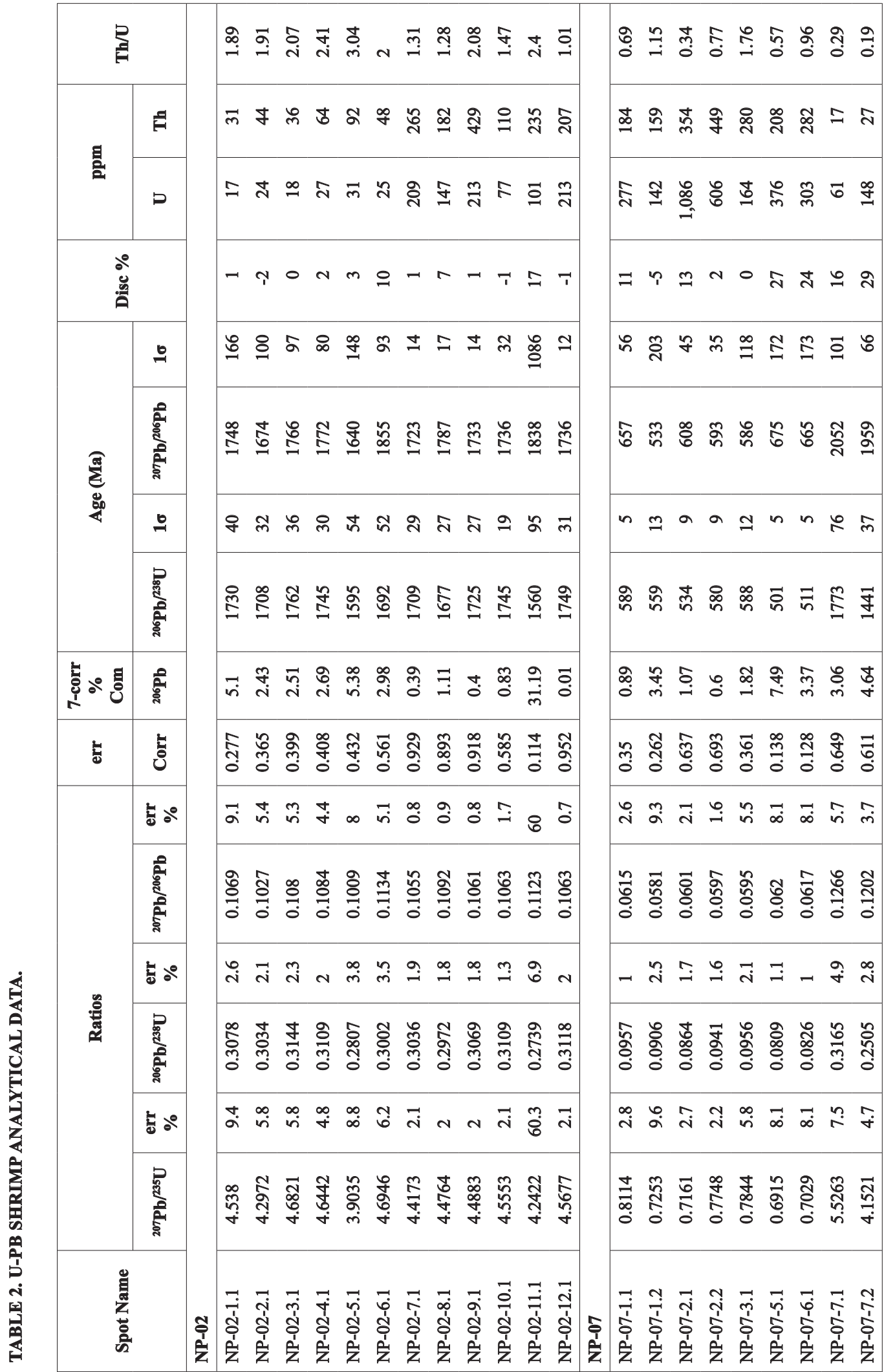




\begin{tabular}{|c|c|c|c|c|c|}
\hline \multicolumn{2}{|l|}{$\stackrel{1}{s}$} & & 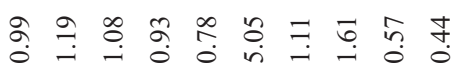 & & 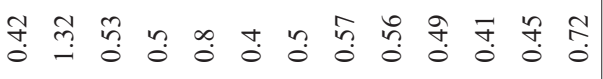 \\
\hline & $\cong$ & & 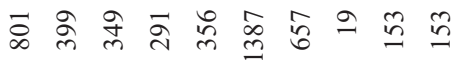 & & 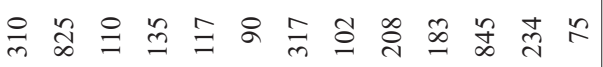 \\
\hline & $\triangleright$ & & 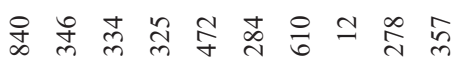 & & 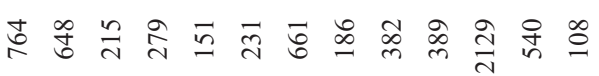 \\
\hline \multicolumn{2}{|l|}{$\begin{array}{l}\dot{0} \\
\ddot{\omega}\end{array}$} & & $f 0=\underset{m}{f} N F 0 \nvdash m$ & & 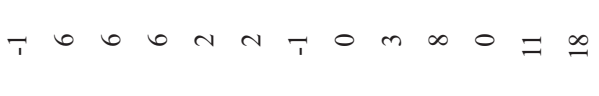 \\
\hline \multirow{4}{*}{ 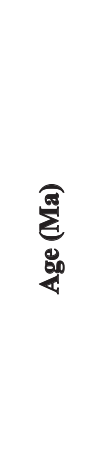 } & $\stackrel{0}{-}$ & & ๙े & & 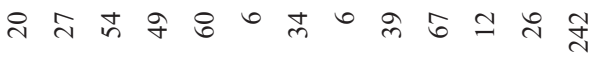 \\
\hline & 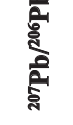 & & 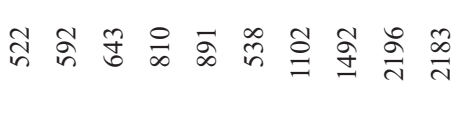 & & \& \\
\hline & $\stackrel{0}{-}$ & & 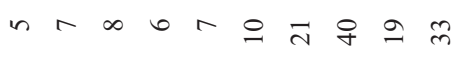 & & 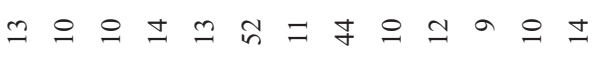 \\
\hline & 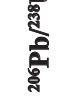 & & 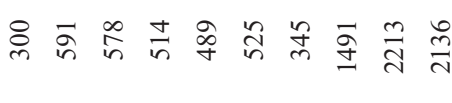 & & 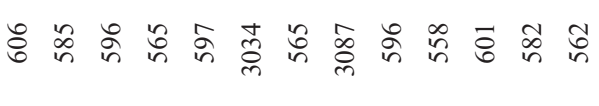 \\
\hline 竞 & : & & 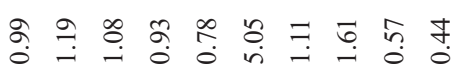 & & 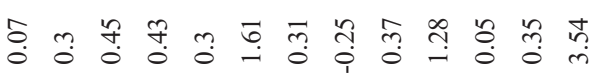 \\
\hline 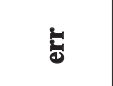 & ठ் & & 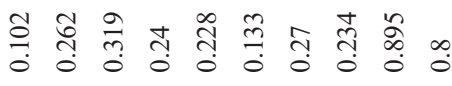 & & 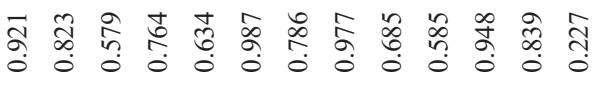 \\
\hline & $\overbrace{}^{\circ}$ & & 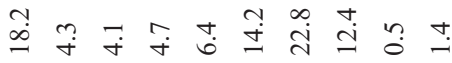 & & 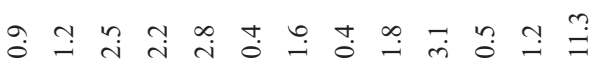 \\
\hline & 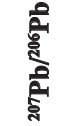 & & 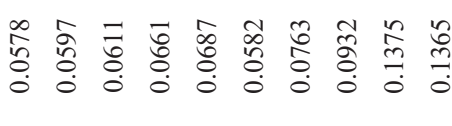 & & 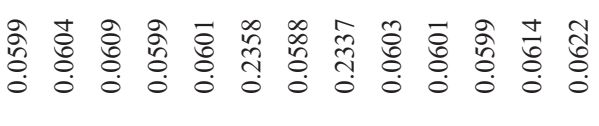 \\
\hline & $\leftrightarrows 0^{\circ}$ & & 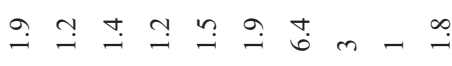 & & 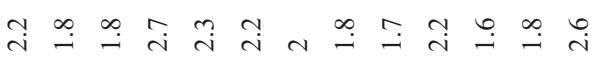 \\
\hline & 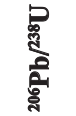 & & 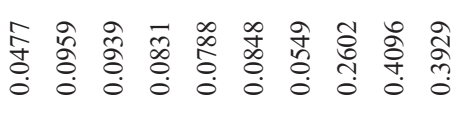 & & 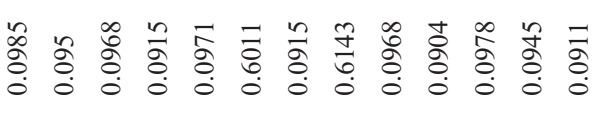 \\
\hline & to & & 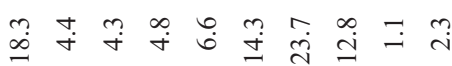 & & 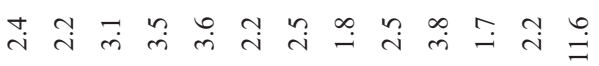 \\
\hline & 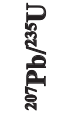 & & 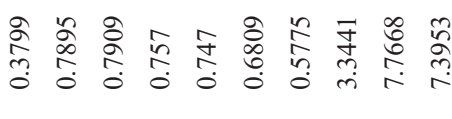 & & 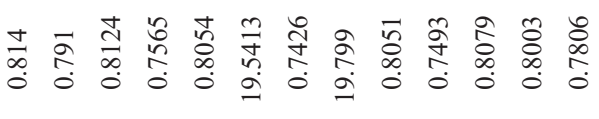 \\
\hline \multicolumn{2}{|l|}{ 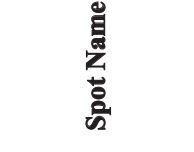 } & $\begin{array}{l}\infty \\
\substack{1 \\
1 \\
z \\
z}\end{array}$ & 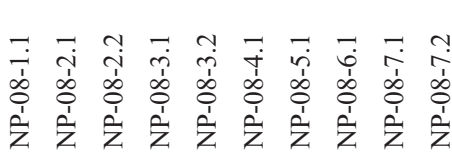 & 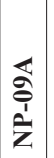 & 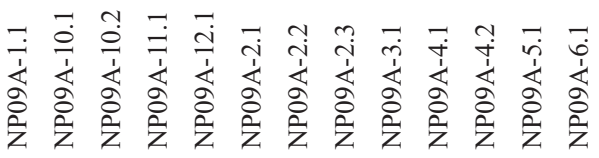 \\
\hline
\end{tabular}




\begin{tabular}{|c|c|c|c|c|c|c|}
\hline \multicolumn{2}{|l|}{$\stackrel{1}{p}$} & 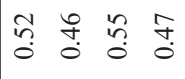 & & 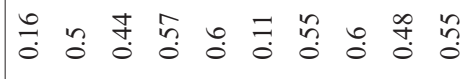 & & 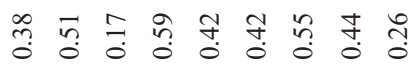 \\
\hline \multirow{2}{*}{ 气̆ } & $\approx$ & $\stackrel{\infty}{\infty} \approx n$ & & 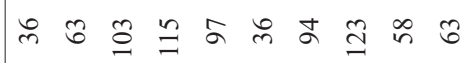 & & 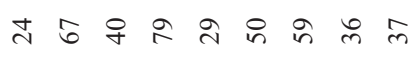 \\
\hline & D & 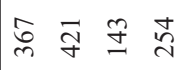 & & 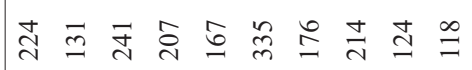 & & 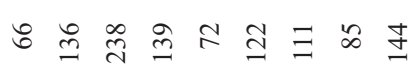 \\
\hline \multicolumn{2}{|l|}{ 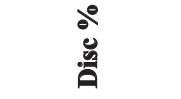 } & $\because \ddot{c} \pm+$ & & $\infty-00+0-t+n$ & & $0 \mathrm{n}$ in $m \mathrm{~h}-\mathrm{h} m$ \\
\hline \multirow{4}{*}{ 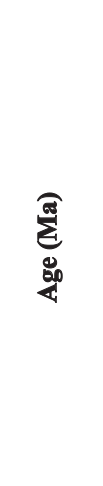 } & $\stackrel{0}{\circ}$ & $\underset{m}{\infty} \stackrel{2}{\infty} \approx \tilde{6}$ & & $0 \wedge n \pm r \underline{-} \sim 0 \infty \infty$ & & $= \pm 0 r \propto \infty \cong a$ \\
\hline & 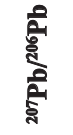 & 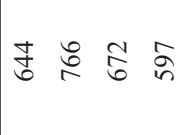 & & 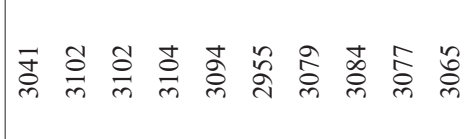 & & 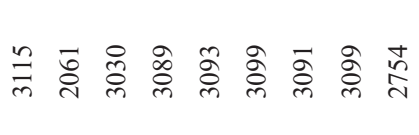 \\
\hline & $\stackrel{0}{-}$ & $\cong \simeq \cong$ & & in $q$ q & & in \\
\hline & 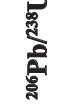 & 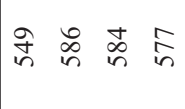 & & 會 & & 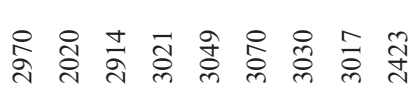 \\
\hline قُ & : & 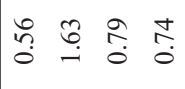 & & 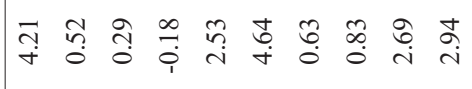 & & 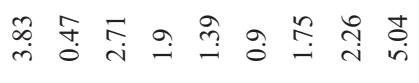 \\
\hline 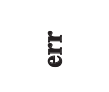 & uे & 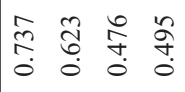 & & 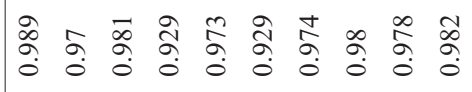 & & 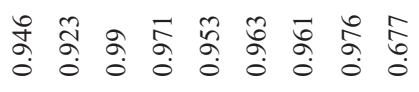 \\
\hline \multirow{6}{*}{ 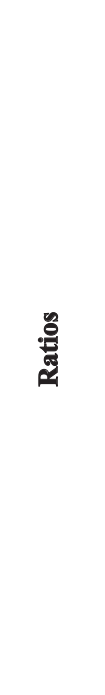 } & $5 \circ$ & $\stackrel{\infty}{\rightarrow} \underset{i}{\stackrel{\nabla}{r}} \overrightarrow{\dot{m}}$ & & 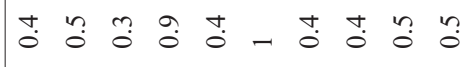 & & 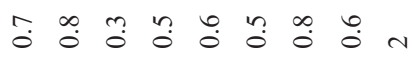 \\
\hline & 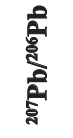 & 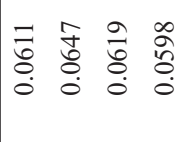 & & 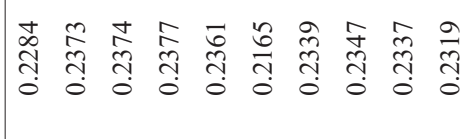 & & 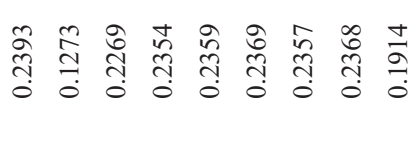 \\
\hline & $\leftrightarrows 0^{\circ}$ & $\stackrel{\leftrightarrow}{-} \vec{i} \stackrel{\infty}{-} \stackrel{\infty}{-}$ & & 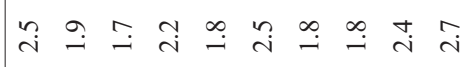 & & 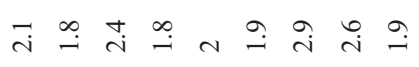 \\
\hline & 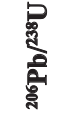 & 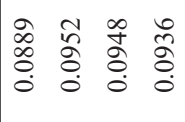 & & 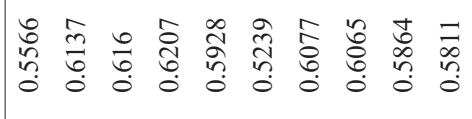 & & 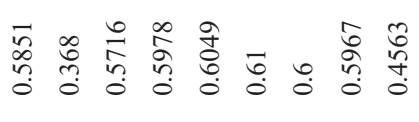 \\
\hline & $5 \circ$ & 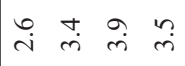 & & 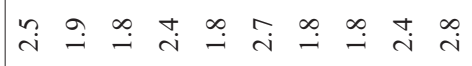 & & 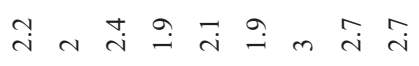 \\
\hline & 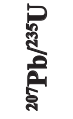 & 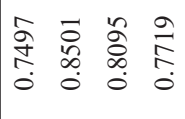 & & 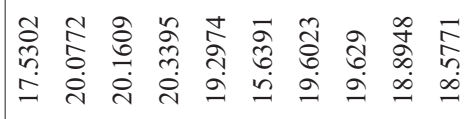 & & 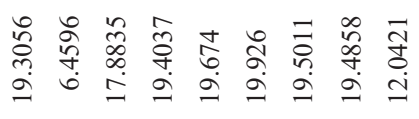 \\
\hline \multicolumn{2}{|c|}{$\bar{z}$} & 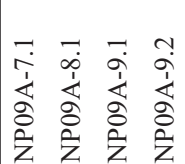 & $\begin{array}{l}\vec{m} \\
\hat{a} \\
\grave{z}\end{array}$ & 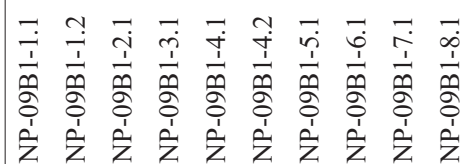 & $\mid$ & 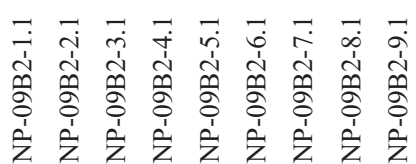 \\
\hline
\end{tabular}




\begin{tabular}{|c|c|c|c|c|c|c|}
\hline \multicolumn{2}{|l|}{$\stackrel{2+2}{p}$} & 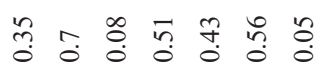 & & 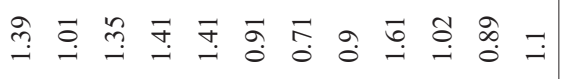 & & 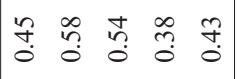 \\
\hline \multirow{2}{*}{ 言 } & $\cong$ & $\approx \infty$ d & & 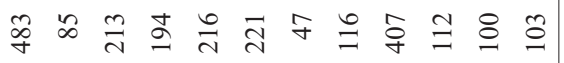 & & 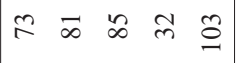 \\
\hline & D & 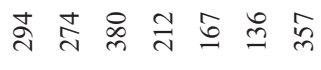 & & 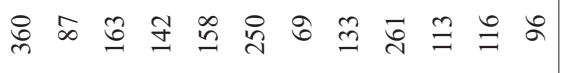 & & 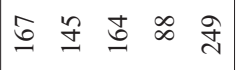 \\
\hline \multicolumn{2}{|c|}{$\stackrel{\circ}{\breve{g}}$} & 0 o $9+t+m$ & & กิ - กิ & & $\begin{array}{lllll}n & 7 & 4 & 4 & 1\end{array}$ \\
\hline \multirow{4}{*}{ 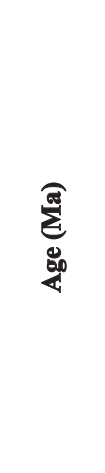 } & $\stackrel{0}{-}$ & $\Rightarrow$ n & & 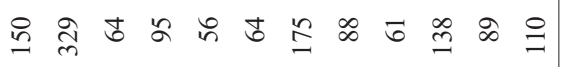 & & $\infty \quad \infty \underset{\sim}{\infty} \simeq 0$ \\
\hline & 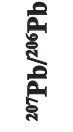 & 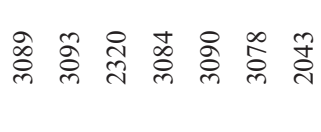 & & 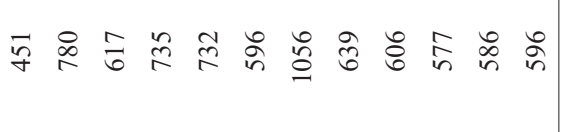 & & 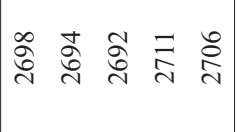 \\
\hline & $\stackrel{0}{=}$ & 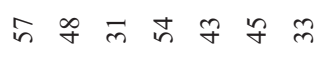 & & $\simeq \subseteq \simeq+0 \subseteq n \pm \simeq=0 \circ$ & & f \\
\hline & 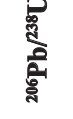 & 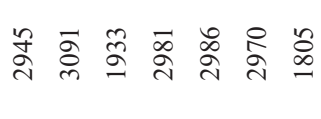 & & 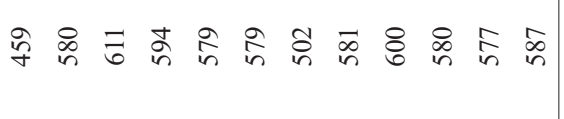 & & 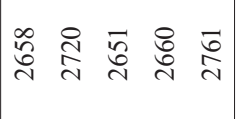 \\
\hline 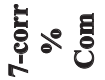 & 言 & 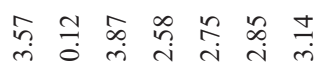 & & 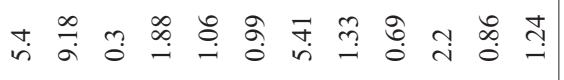 & & 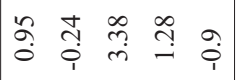 \\
\hline t & ن் & 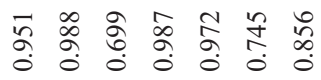 & & 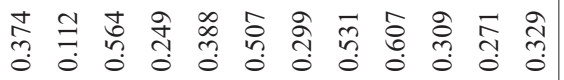 & & $\left|\begin{array}{lllll}0 & 0 & 0 & 0 & 0 \\
\circ & 0 & 0 & 0 \\
0 & 0 & 0 & 0 \\
0 & 0 & 0 & 0 & 0\end{array}\right|$ \\
\hline \multirow{6}{*}{ 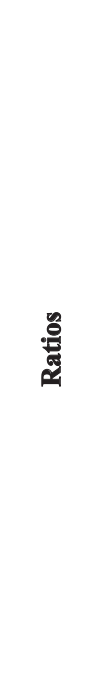 } & $\frac{t}{2} \circ^{\circ}$ & 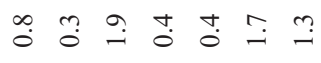 & & 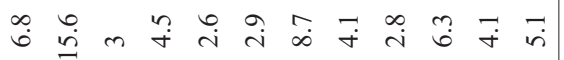 & & 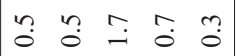 \\
\hline & 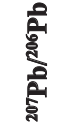 & 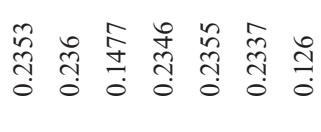 & & 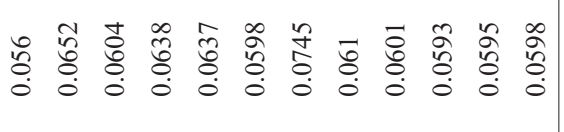 & & $\left|\begin{array}{ccccc}n & 1 & 0 & 0 & \infty \\
\infty & \infty & 0 & \infty \\
0 & \stackrel{0}{0} & \infty & \infty \\
0 & 0 & 0 & 0 & 0 \\
0 & 0\end{array}\right|$ \\
\hline & $\pm \circ$ & 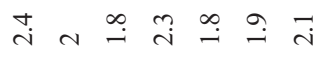 & & 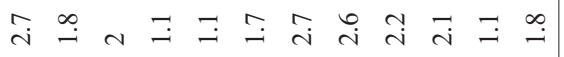 & & $\stackrel{9}{-} \stackrel{n}{-} \stackrel{\infty}{\rightarrow} \stackrel{\infty}{\rightarrow}$ \\
\hline & 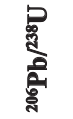 & 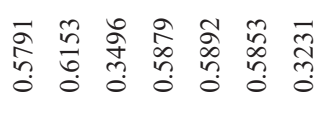 & & 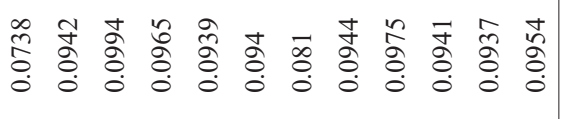 & & 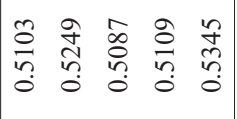 \\
\hline & $5 \circ$ & 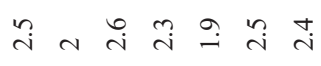 & & 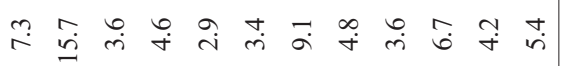 & & 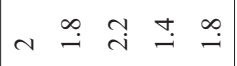 \\
\hline & 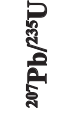 & 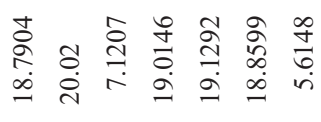 & & 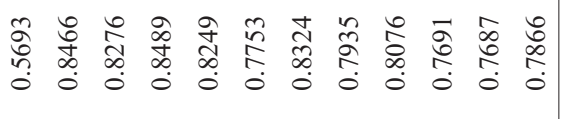 & & 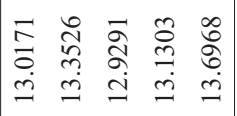 \\
\hline \multicolumn{2}{|c|}{$\begin{array}{l}\sum_{2}^{\pi} \\
\text { aे } \\
\text { के }\end{array}$} & 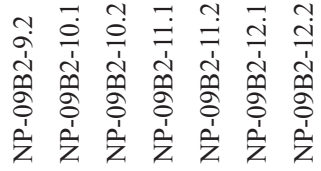 & $\frac{0}{1}$ & 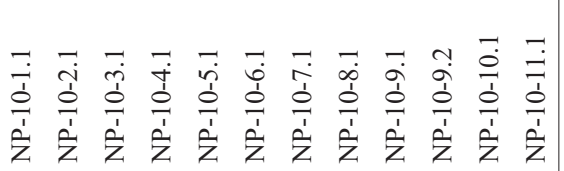 & $\begin{array}{l}\overline{1} \\
\overline{\mathrm{z}}\end{array}$ & 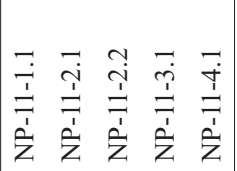 \\
\hline
\end{tabular}




\begin{tabular}{|c|c|c|c|}
\hline \multicolumn{2}{|c|}{$\stackrel{?}{E}$} & 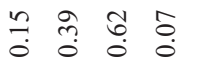 & $\begin{array}{ccc}n & n & n \\
0 & n & 0\end{array}$ \\
\hline \multirow{2}{*}{ 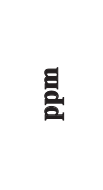 } & $\Xi$ & $\pm f \& \circlearrowright$ & $\cong \approx \sigma$ \\
\hline & $D$ & 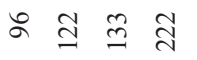 & $\stackrel{ }{\stackrel{I}{I}} \vec{i}$ \\
\hline \multicolumn{2}{|c|}{$\begin{array}{l}\stackrel{0}{\circ} \\
\ddot{y} \\
\ddot{\theta}\end{array}$} & $=r r N$ & T 4 \\
\hline \multirow{4}{*}{ 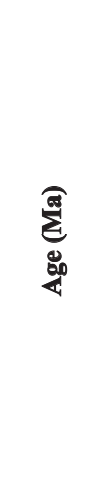 } & $\underline{0}$ & $\infty \infty^{\infty} \infty$ & $0 \cong 0$ \\
\hline & 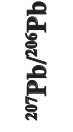 & 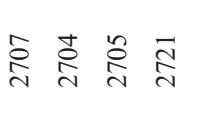 & 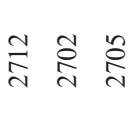 \\
\hline & $\stackrel{0}{-}$ & 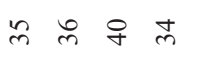 & $m \approx q$ \\
\hline & 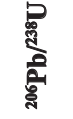 & 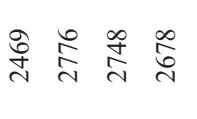 & 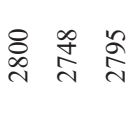 \\
\hline 这。 & : & $m=\overrightarrow{0} \sigma$ & 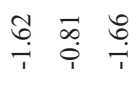 \\
\hline t & uั & 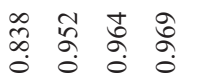 & 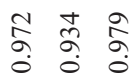 \\
\hline \multirow{6}{*}{ 气ू̆ } & $50^{\circ}$ & $\exists \begin{array}{llll}n & n & n & 0\end{array}$ & $\stackrel{t}{\circ}: \stackrel{0}{0}:$ \\
\hline & 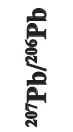 & 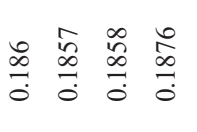 & $\begin{array}{lll}n & + & \infty \\
\infty & 0 \\
\infty & \infty & \infty \\
0 & 0 & 0 \\
0 & 0 & 0\end{array}$ \\
\hline & 50 & I. $\stackrel{\circ}{-} \stackrel{\infty}{-} \stackrel{\circ}{-}$ & $\because \stackrel{0}{=}$ \\
\hline & 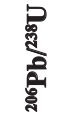 & 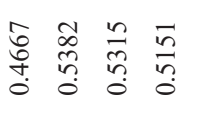 & 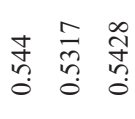 \\
\hline & $5 \circ$ & $\alpha \stackrel{\circ}{-} \stackrel{\circ}{-}$ & $\because=\stackrel{\infty}{=}$ \\
\hline & 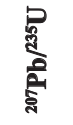 & 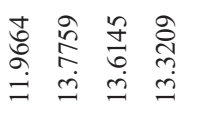 & 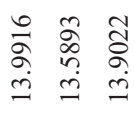 \\
\hline \multicolumn{2}{|c|}{ 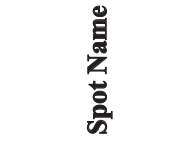 } & 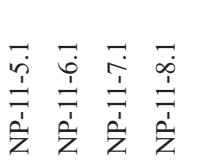 & 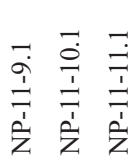 \\
\hline
\end{tabular}

analyzed in their core and rim (Fig. 5b). Both, core and rim, yielded similar ages. Figure 6 shows the concordant age of $584 \pm 8$ Ma obtained from three out of the six grains (highly discordant data was discarded). Based on zircon morphology and $\mathrm{Th} / \mathrm{U}$ ratios $(>0.34)$, this result is considered a good estimate for its crystallization age. Zircon \#7 is an inherited xenocrystal, with a rather imprecise Rhyacian age of about $2100 \mathrm{Ma}$.

\subsection{Sample NP-08-Cerro Chato granite}

This sample was collected at the western margin of the Cerro Chato granite, in the central part of the Nico Pérez Terrane; also, it intrudes the ValentinesRivera Granulitic Complex (Fig. 2). It is a fine to coarse-grained granite, sometimes porphyric, with large zoned feldspars, plagioclase, quartz, muscovite and biotite. Zircon, apatite, and epidote are also present as accessory minerals. Locally, this granite presents mylonitic foliation.

A total of ten spots were analysed out of seven zircon crystals (Table 2 and Fig. 5c). Zircon grains are prismatic, rounded, or fragmented, and show cores that are either light or dark homogeneous or zoned. Homogeneous dark rims are observed. Most of the analytical results show high discordance, and $\mathrm{Pb}$ loss is observed (Fig. 7). A concordant age of $591 \pm 7 \mathrm{Ma}$ is obtained using two zircon grains (highly discordant data was discarded), probably it represents the crystallization age of this pluton. Two inherited crystals yielded Paleoproterozoic ( $c a .2 .1 \mathrm{Ga}$ ) and Mesoproterozoic (ca. 1.5 Ga) ages, respectively.

\subsection{Samples NP-09A, NP-09B1 and NP-09B2- Mylonitic granites within the Cueva del Tigre shear zone}

These samples were taken at an outcrop located within the Cueva de la Tigre shear zone in the contact of two deformed granitoids, affected by the shear zone (Fig. 2). Sample NP-09A is a granitoid specimen, classified in the field as protomylonitc granite, presenting quartz ribbons and quartz crystals with subgrain rotation and bulging recrystallization. Sample NP-09B is a foliated granitoid, showing a banded fabric, splitted into a lighter specimen (NP-09B1) and a darker one (NP-09B2) with some more biotite and less plagioclase due to mineral segregation. Petrographically, it is composed of 


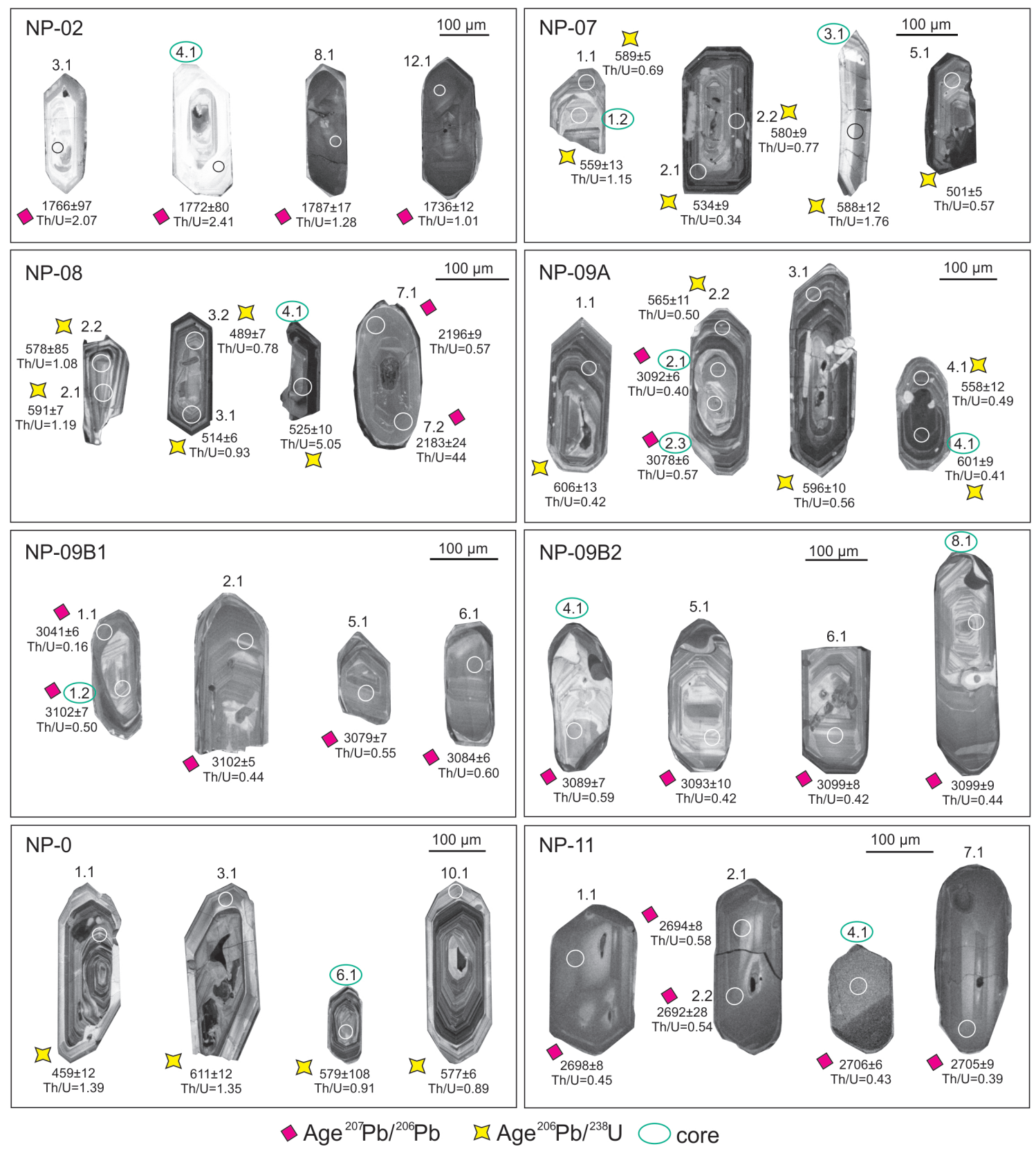

FIG. 5. The cathodoluminescence images of representative analyzed zircons.

quartz $+\mathrm{K}$-feldspar+plagioclase+biotite and chlorite. K-feldspar crystals also show bulging recrystallization and undulose extinction.

Zircon grains in sample NP-09A are prismatic with oscillatory zoning, and some of them show xenocrystal cores. Twelve zircon crystals were analyzed in 17 spots. Figure 10 shows a concordant age of $588.0 \pm 6.1 \mathrm{Ma}$ obtained from 10 spots out of 17 (discordant data was discarded), interpreted as the crystallization age. Xenocrystal core yielded Mesoarchean ages (\#2.1 and \#2.3 in Table 2). Besides, all the analyzed crystals of this sample exhibited $\mathrm{Th} / \mathrm{U}$ ratios ( 0.40 to 1.32 ) typical of magmatic rocks. 


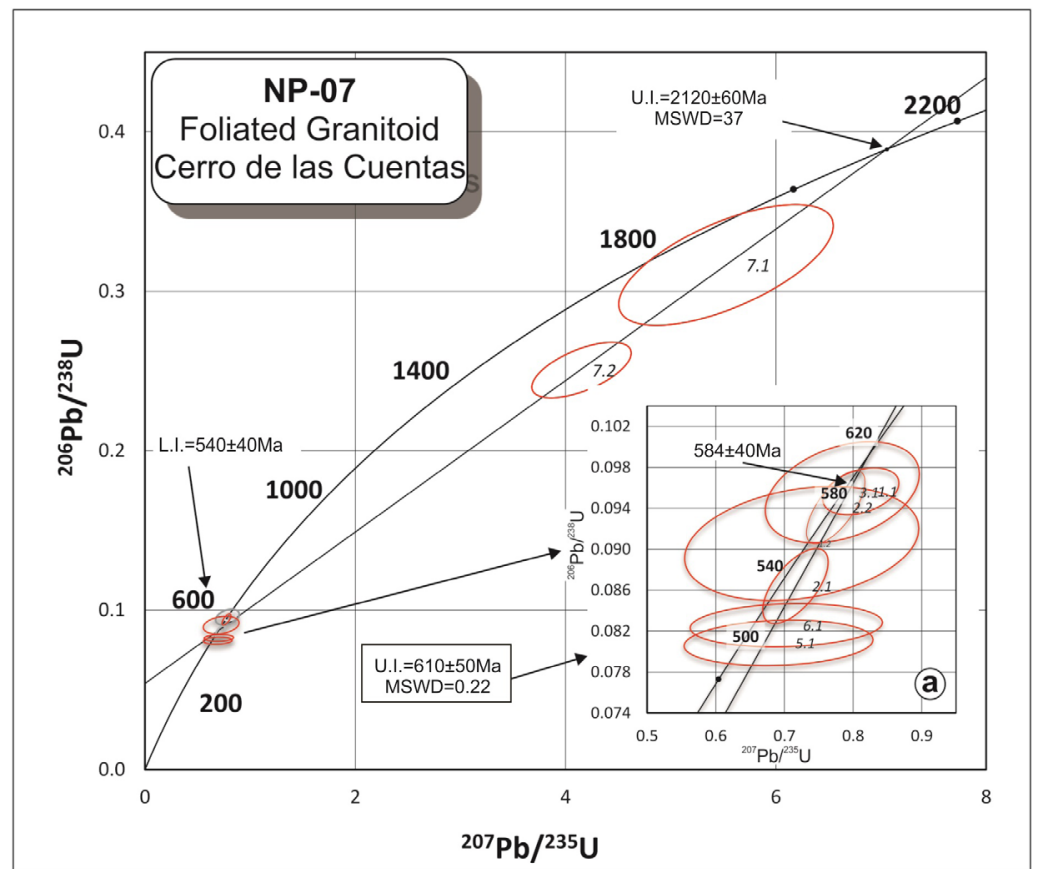

FIG. 6. U-Pb SHRIMP Concordia Diagram for the Cerro de las Cuentas granite (NP-07); a) ellipses indicate the analyses used for the age calculation. Error ellipses are at $95 \%$ confidence level.

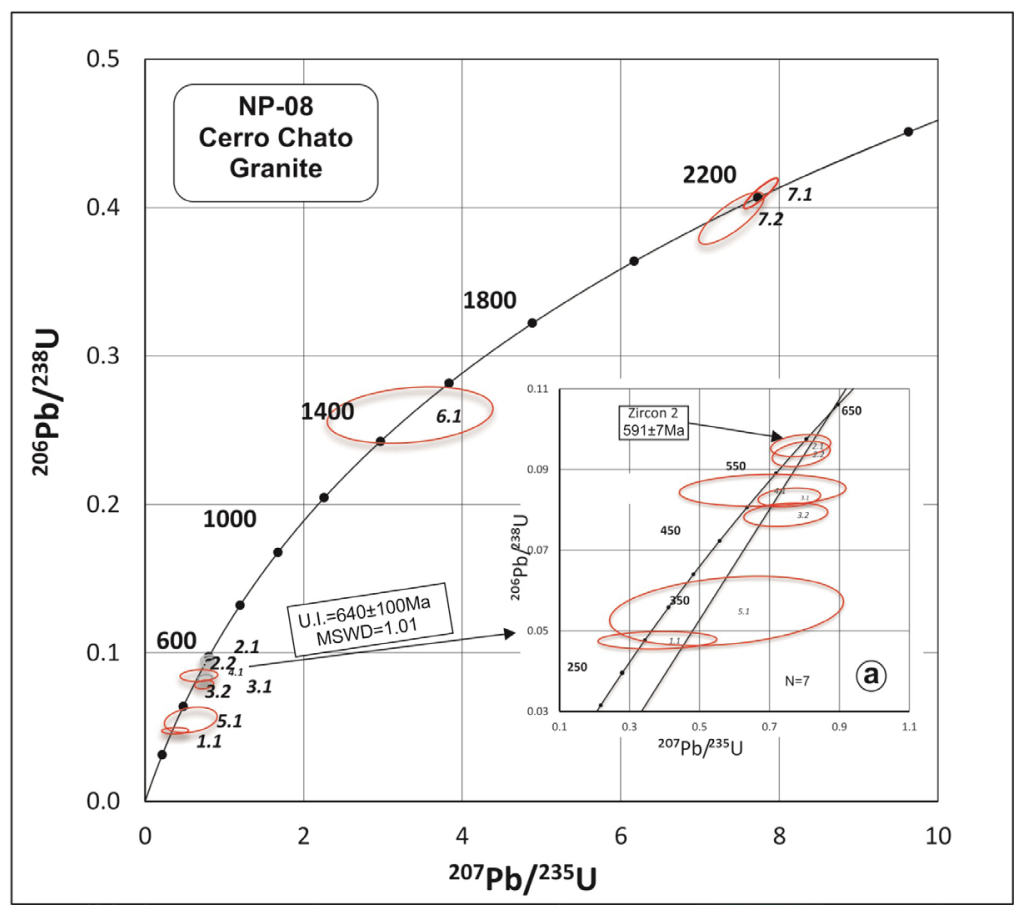

FIG. 7. U-Pb SHRIMP Concordia Diagram for the Cerro Chato granite (NP-08); a) ellipses indicate the analyses used for the age calculation. Error ellipses are at $95 \%$ confidence level. 


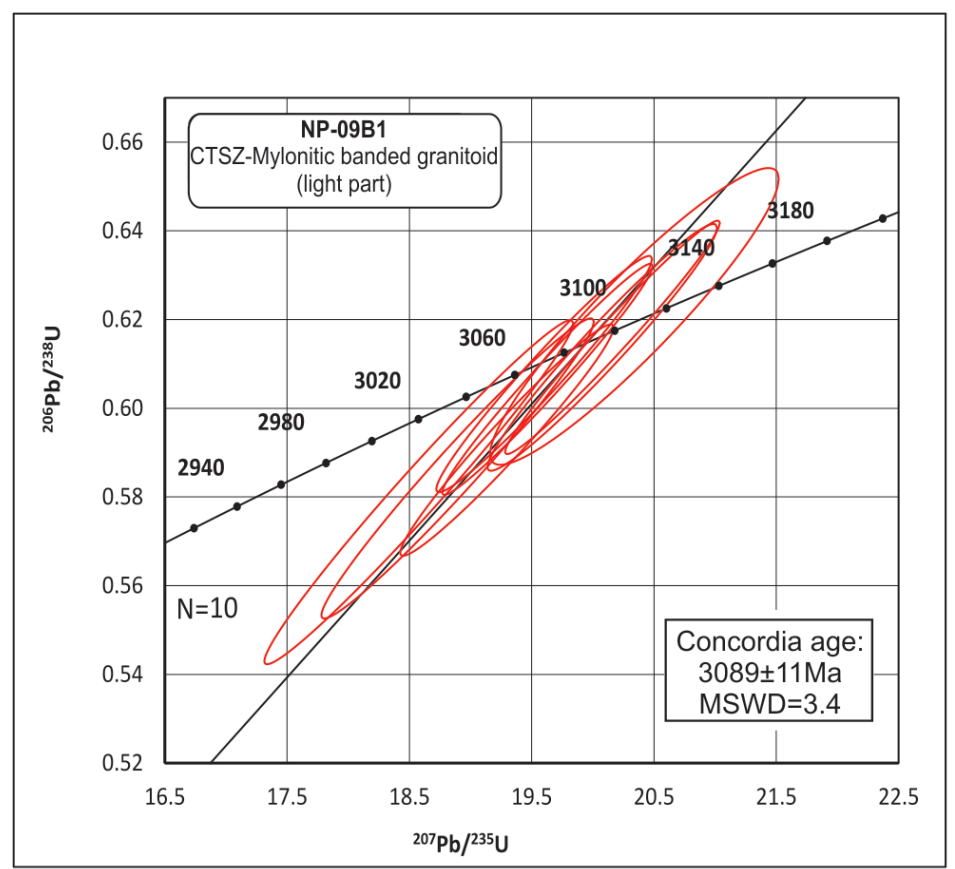

FIG. 8. U-Pb SHRIMP Concordia Diagram for Mylonitic granite form Cueva del Tigre shear zone (NP-09B1); ellipses indicate the analyses used for the age calculation. Error ellipses are at $95 \%$ confidence level.

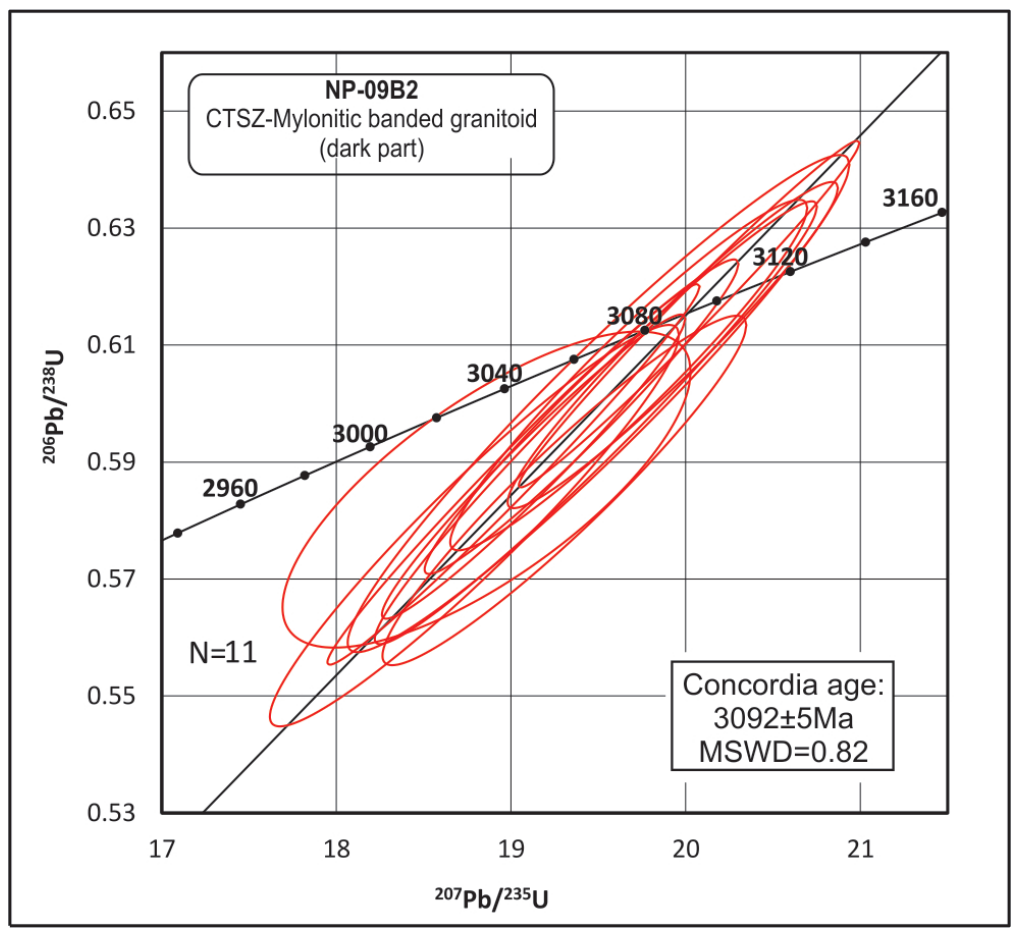

FIG. 9. U-Pb SHRIMP Concordia Diagram for Mylonitic granite form Cueva del Tigre shear zone (NP-09B2); ellipses indicate the analyses used for the age calculation. Error ellipses are at $95 \%$ confidence level. 


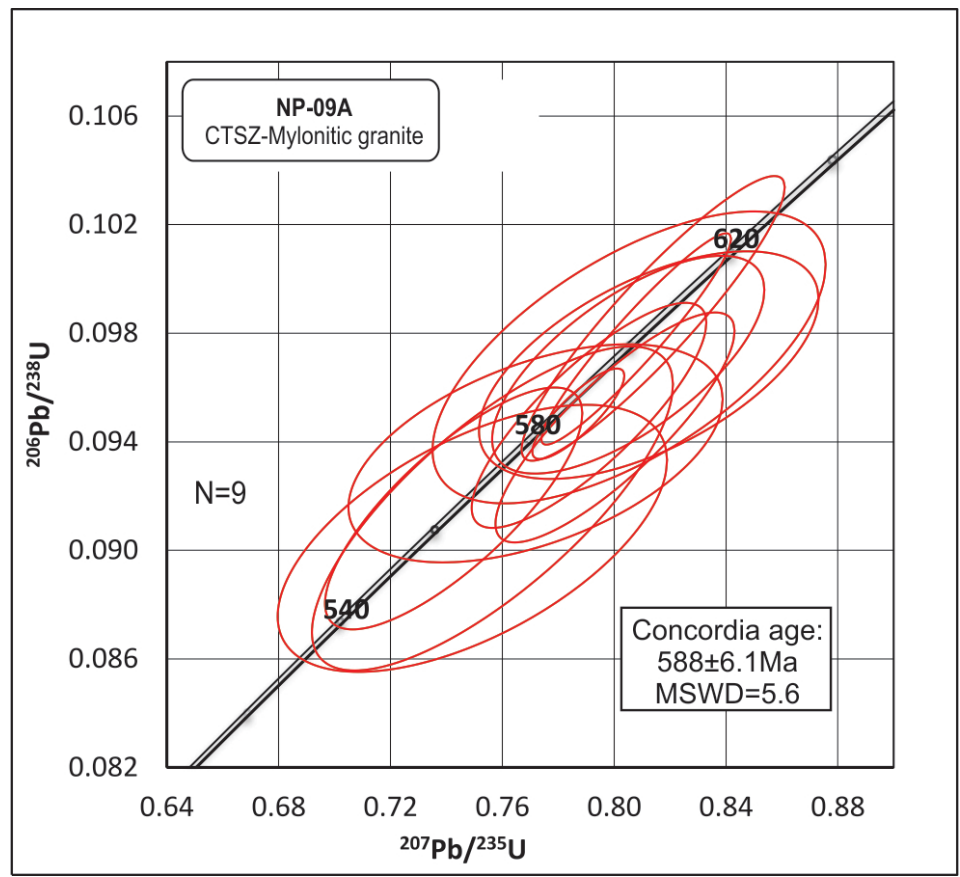

FIG. 10. U-Pb SHRIMP Concordia Diagram for Mylonitic granite form Cueva del Tigre shear zone (NP-09A); ellipses indicate the analyses used for the age calculation. Error ellipses are at $95 \%$ confidence level.

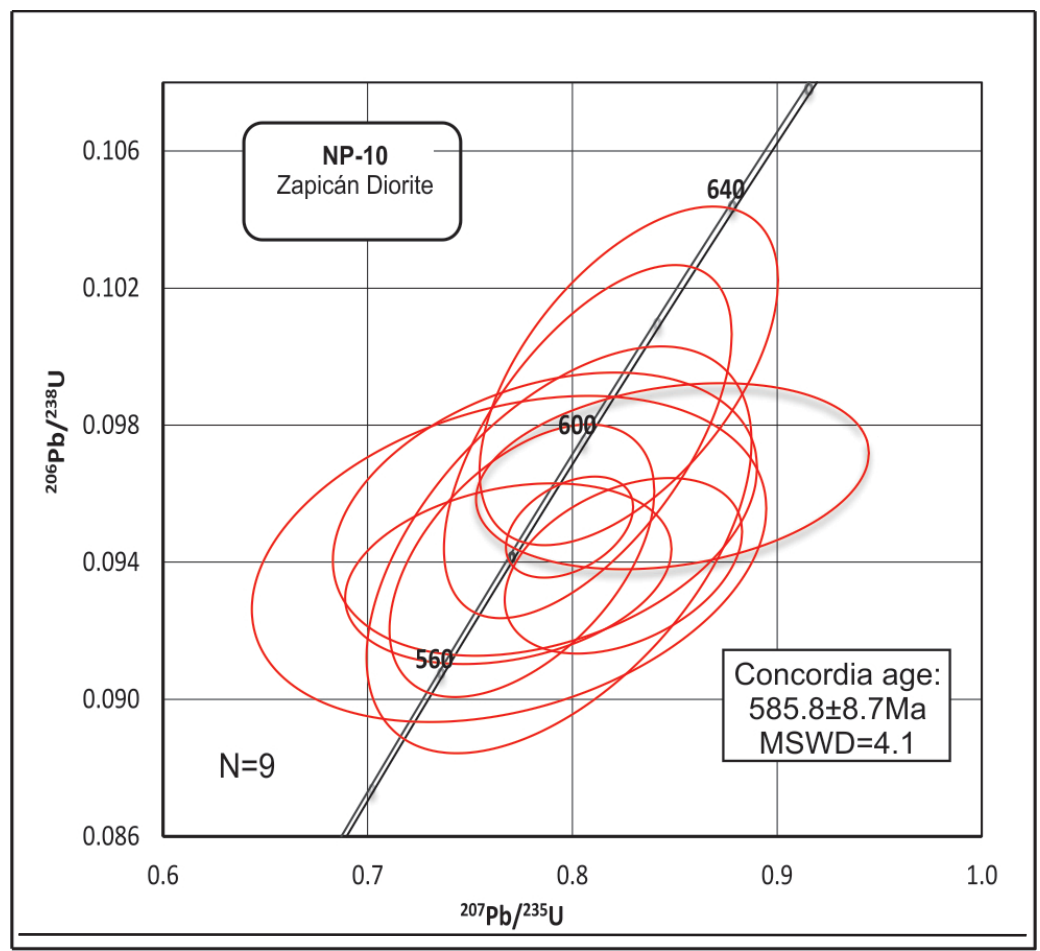

FIG. 11. U-Pb SHRIMP Concordia Diagram for La China Complex (NP-10); ellipses indicate the analyses used for the age calculation. Error ellipses are at $95 \%$ confidence level. 


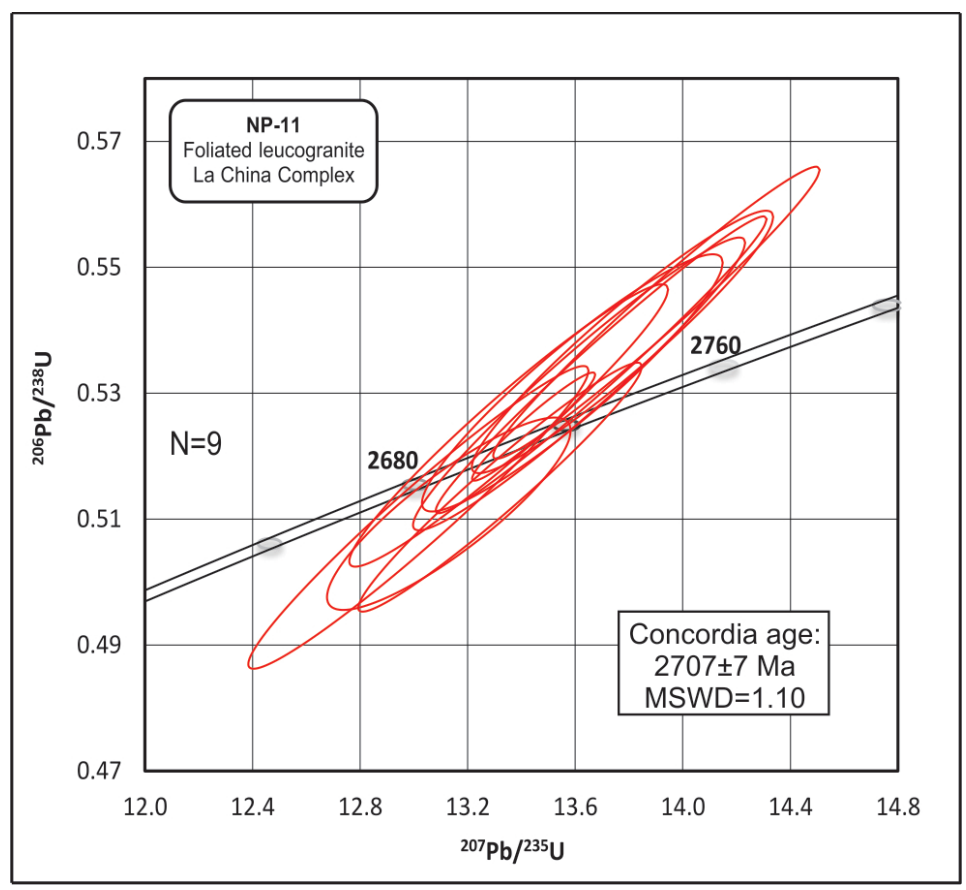

FIG. 12. U-Pb SHRIMP Concordia Diagram for La China Complex (NP-11); ellipses indicate the analyses used for the age calculation. Error ellipses are at $95 \%$ confidence level.

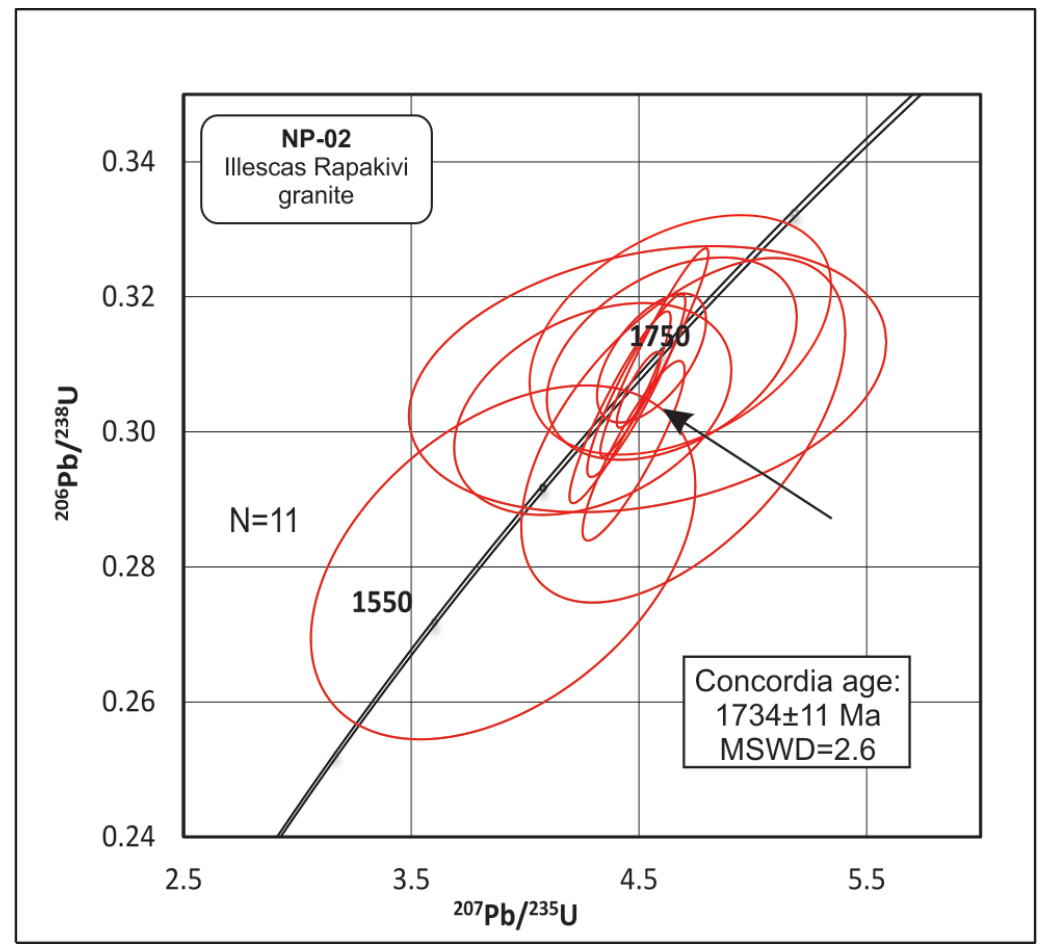

FIG. 13. U-Pb SHRIMP Concordia Diagram for Illescas rapakivi granite (NP-02); ellipses indicate the analyses used for the age calculation. Error ellipses are at 95\% confidence level. 
Zircon grains in samples NP-09B1 and NP-09B2 are also prismatic with oscillatory zoning cut off by areas of re-homogenization and with $\mathrm{Th} / \mathrm{U}$ ratios between 0.17 to 0.70 . Eight grains of the NP-09B1 sample yielded a concordant age of $3089 \pm 11 \mathrm{Ma}$ (10 spots) (Fig. 8). In the case of sample NP-09B2, 16 spots were measured in 12 zircon crystals. A concordant age of $3092 \pm 5 \mathrm{Ma}$ (Fig. 9) were obtained from 11 out of 16 spots (highly discordant data was discarded). Both samples show almost identical ages interpreted here as the crystallization age.

\subsection{Sample NP-10-Deformed Zapicán diorite}

This sample was also collected close to the Cueva del Tigre shear zone, not far from outcrop NP-09 (Fig. 2). The rock corresponds to the Zapicán diorite, composed by plagioclase+amphibole (+quartz $+\mathrm{K}-$ feldspar+biotite). It is locally foliated, presenting some bulging recrystallization and undulose extinction in quartz crystals.

Eleven zircon crystals were analyzed (Table 2). Zircon grains are generally prismatic with oscillatory zoning; also, some grains contain areas of re-homogenization and local recrystallization (Fig. 5). $\mathrm{Th} / \mathrm{U}$ ratios of the analyzed zircon grains range from 0.71 to 1.61. Nine out of twelve spots were concordant and produced a very robust age of $585.8 \pm 8.7 \mathrm{Ma}$ (Fig. 11) interpreted as the time of rock crystallization.

\subsection{Sample NP-11-Foliated leucogranite of the La China Complex}

This sample was collected in the southern exposure of the La China Complex and corresponds to a foliated biotitic leucogranite (Fig. 2). Petrographically, it is made up of plagioclase, quartz and perthitic microcline as the predominant minerals, muscovite and biotite are arranged parallel to the foliation.

Zircon grains are generally prismatic, stubby, with areas of re-homogenization (Fig. 5) and contain $\mathrm{Th} / \mathrm{U}$ ratios between 0.07 and 0.62 . Twelve spots from eleven zircon crystals were analyzed yielding a concordant age of $2707 \pm 7 \mathrm{Ma}$ (Fig. 12) interpreted as the crystallization age.

\subsection{Sample NP-02-Illescas rapakivi granite}

The analyzed sample was collected in the central part of the Illescas rapakivi granite (Fig. 2) and corresponds to an inequigranular, medium grained granitoid, composed of quartz $+\mathrm{K}$-feldspar \pm plagioclase \pm amphibole \pm biotite \pm titanite \pm zircon \pm apatite. Deformation (undulose extinction and bulging recrystallization) is observed in quartz and K-feldspars, and perthites are present. Profuse sericitic alteration is observed.

Twelve zircon crystals were analyzed, being prismatic, either dark or light, with oscillatory zoning, and $\mathrm{Th} / \mathrm{U}$ ratios from 1.10 to 2.40 . Eleven concordant spots yielded a robust age of $1734 \pm 11 \mathrm{Ma}$, shown in figure 13, and interpreted as the crystallization age.

\section{Discussion}

\subsection{Archean ages in the Nico Pérez Terrane}

When considering the whole geochronologic data, both old and new data presented here, relevant improvements for the understanding of the tectonic evolution of the Nico Pérez Terrane are achieved. Archean age of metaigneous rocks from the Pavas Block, formerly reported by Hartmann et al. (2001), was confirmed by three samples of La China Complex. Two of them are from a mylonitic granite (NP-09B) affected by the Cueva del Tigre shear zone. These samples show Mesoarchean ages whose results are identical within experimental error (NP-09B1=3,089 $\pm 11 \mathrm{Ma}$ and NP-09B2=3092 $\pm 5 \mathrm{Ma}$ ), suggesting a magmatic event at that time. The third sample from Pavas Block is a foliated leucogranite yielding an age of $2707 \pm 7 \mathrm{Ma}$ assumed as magmatic. Thus, the ages of magmatism (ca. 3-2.7 Ga; Table 1) and metamorphism (ca. $2.7 \mathrm{Ga}$; Table 1) reported by different authors (Hartmann et al., 2001; Gaucher et al., 2014a; and references therein), as well as those carried out in this study, can be gathered into two groups: Mesoarchean and Neoarchean.

Moreover, the detrital zircon grain ages reported in the literature for Las Tetas Complex and Valentines-Rivera Granulitic Complex (Valentines Formation), suggest the existence of Paleo to Mesoarchean source areas. This is also supported by Lu-Hf data obtained by Oriolo et al. (2016a) for this area. The age of $3.4 \mathrm{Ga}$ reported by Hartmann et al. (2001) could correspond to a Paleoarchaean crust. Then, in tectonomagmatic events around 3.1 Ga formed the tonalitic rocks and around $2.7 \mathrm{Ga}$ occured magmatism and metamorphism 
at medium-high grade (Hartmann et al., 2001; Gaucher et al., 2011).

\subsection{The Illescas Batholith: tectonic significance}

The robust U-Pb Statherian zircon age of $1734 \pm$ 11 Ma obtained here for the Illescas Batholith, which is intrusive into the western part of the Valentines Block, is very similar to the ages indicated previously by Bossi and Campal (1992), Campal and Schipilov (1995) and Oriolo et al. (2019). This batholith has been considered related to an extensional episode within the Nico Pérez Terrane, possibly genetically coeval with the extensional event that originated the important Florida dike swarm in the Piedra Alta Terrane located immediately west of the Sarandí del Yí shear zone (Gaucher and Blanco, 2014). In this framework, the Illescas Batholith and the Florida dike swarm could represent the break-up or widespread extension of the Atlantica supercontinent or the Statherian taphrogenic episode (Brito Neves et al., 1995; Gaucher and Blanco, 2014). The Sarandí del Yí shear zone, is located between these two units, along which some horizontal displacement may have taken place (Bossi and Campal, 1992).

Scarce and incomplete set of geochemical data (only two samples) for the Illescas Batholith presented by Gaucher and Blanco (2014) indicate a within plate anorogenic environment. However, Oriolo et al. (2019) based on a complete set of geochemical data (seven samples), suggested a post-collisional/ post-orogenic setting. According to the available ages for the Nico Pérez Terrane, this suggestion is debatable, since the previous recorded orogenic event happened $c a$. $200 \mathrm{Ma}$ before. Therefore, the most realistic hypotesis is an anorogenic setting. In addition, the alkali-calcic signature mentioned by Oriolo et al. (2019) could be related to typical crustal contamination.

\subsection{Neoproterozoic magmatism in the Nico Pérez Terrane}

Neoproterozoic crystallization ages are recorded in four intrusive granitoids in Valentines-Rivera Granulitic Complex and in La China Complex. The mylonitic granite (sample NP-09A) affected by Cueva del Tigre shear zone, yielded an Ediacaran age of $588 \pm 6.1 \mathrm{Ma}$. This sample contains an inherited zircon crystal with a xenocrystal core of $3089 \pm 13 \mathrm{Ma}$, identical to the neighbor sample NP-09B. This feature also suggests a Mesoarchean age for the protolith of this unit. In addition, it is confirmed the Ediacaran age for the Zapicán diorite (585.8 \pm 5.7 ), even though it is younger than the previous report made by Oriolo et al. (2016a) of $610 \pm 2.5 \mathrm{Ma}$.

In the Valentines Block, a few Rhyacian inherited zircon xenocrystals with ages between 2200 and $2100 \mathrm{Ma}$ were obtained in Cerro de Las Cuentas and Cerro Chato Ediacaran granitic plutons (NP-07 and NP-08, respectively). This evidence confirms previous ages obtained by Santos et al. $(2003,2017)$ and Oriolo et al. (2016a) for the widespread Paleoproterozoic basement rocks. Hence, the obtained results of our work confirm the post-orogenic Ediacaran magmatism that affected both the Valentines and Pavas blocks. These Ediacaran ages are comparable with several data reported in the literature for the Nico Pérez Terrane and the Dom Feliciano Belt (Mallmann et al., 2007; Sánchez Bettucci et al., 2010b; Oriolo et al., 2016a).

The widespread granitoid magmatism of Neoproterozoic age (see Fig. 2) was coupled with the marked structural NE-SW shearing trend (Preciozzi et al., 1979; Sánchez Bettucci et al., 2010b). This structural feature affected the entire terrane and caused important disturbances, as shown by multiple shear zones, mylonitic features and regional foliation (Sánchez Bettucci et al., 2010b). A good example is the Cueva del Tigre shear zone, represented by a group of granitic mylonites, schists and phyllonites at the boundary between the Valentines Block and La China Complex (Hartmann et al., 2001). In addition, extensional tectonics may have been the cause of the formation of restricted sedimentary basins affected by low grade metamorphism over the Valentines Block, such as the meta-sandstones and meta-subarkoses of the Cerro San Francisco Formation and the oolitic and stromatolitic meta-limestones of the Cerros Victoria Formation (Montaña and Sprechmann, 1993), during Neoproterozoic times.

The intrusion of the granitic plutons can be attributed to the influence of the long-term tectonomagmatic episode related to the Brasiliano orogenic cycle. The field work carried out during sampling, allows the recognition of the Retamosa thrust as the eastern limit of the Nico Pérez Terrane against the Dom Feliciano supracrustal sequences (Figs. 1 and 2). This thrust is characterized by NNE general orientation with ESE vergence, being cut 
in the southern portion by a NS normal fault. In the hanging wall Neoproterozoic supracrustal rocks outcrop.

\subsection{Cratonic affinity of the Nico Pérez Terrane}

The relative interaction between the Piedra Alta Terrane and the Nico Pérez Terrane (Fig. 1) has been interpreted in two different ways. According to Mallmann et al. (2007), Sánchez Bettucci et al. (2010a) and Santos et al. $(2017,2019)$ these terranes formed a coherent block, before the collision with the Kalahari Craton, when the Dom Feliciano Belt was formed. Therefore, the Nico Pérez Terrane would correspond to a reworked margin of the Río de la Plata Craton that was intensely deformed during the Brasiliano-Pan-African orogeny and adopted the NNE regional structural trend of the Dom Feliciano Belt. On the other hand, Oyhantçabal et al. (2011) challenged the Nico Pérez Terane affinity with the Río de la Plata Craton based on lithologic, structural, geochronologic, and isotopic data. Oriolo et al. (2016a) and Oyhantçabal et al. (2018) suggested that the Nico Pérez Terrane would be an allochthonous crustal segment, originally part of the Congo Craton, that was accreted to the east (currently coordinates) of Piedra Alta Terrane along the Sarandí del Yi shear zone in Ediacaran times. Although interesting, this proposal is still an unfinished debate.

According to Dragone et al. (2017), the Congo Craton presents negative gravity bouguer anomalies whereas the Río de La Plata Craton, including Nico Pérez Terrane, have positive bouguer anomalies suggesting distinct lithosphere. Also, these authors mentioned that Congo Craton has crustal thicknesses above $40 \mathrm{~km}$, while the Río de la Plata has a thinner and denser crustal thickness around $35 \mathrm{~km}$. Furthermore, Bologna et al. (2019) based on magnetotelluric data concluded that no lithospheric-scale contrast in the electrical resistivity between Valentines Block and Piedra Alta Terrane occurs across the Sarandí del Yí shear zone. In fact, Bologna et al. (2019) mentioned that the overall resistivity structures of the Valentines Block and Piedra Alta Terrane are similar. These authors also suggest these two units were already a single and stable tectonic unit probably since the Paleoproterozoic, inferring that the Sarandí del Yi shear zone should be relatively shallow. In addition, Bologna et al. (2019) suggested that Neoproterozoic magmatism might not affected the mantle root under the Valentines Block, concluding that this block is an extension of the Río de La Plata Craton.

The Piedra Alta Terrane, at the core of the Río de La Plata Craton, presents partially different tectonic evolution, even though Archean ages were recently identified in some of its lithological units (Santos et al., 2017). The influence of the Brasiliano orogenic cycle, which is widespread in the Nico Pérez Terrane, is also recorded in some units of the Piedra Alta Terrane (Río de La Plata Craton) according to Santos et al. (2017). Up to now, the only Ediacaran magmatic body in the Piedra Alta Terrane is the anorogenic La Paz granite, with an age of $587 \mathrm{Ma}$ (Cingolani et al., 2012).

In a general way, the mainly Rhyacian units of the Piedra Alta Terrane, located within Arroyo Grande and San José belts, were originated in a magmatic arc setting, where tonalites, trondhjemites and granites predominate (Sánchez Bettucci et al., 2010a and references therein). Nevertheles, in the Valentines Block (Nico Pérez Terrane) Rhyacian ages are present being the different units strongly deformed and metamorphosed by the Brasiliano orogenic cycle. A similar relation is encountered to the north, within the Rivera Block.

The exposed crustal level at the present surface in the Piedra Alta Terrane and the Nico Pérez Terrane seems to be different, considering the presence of a great amount of granulitic rocks in the Nico Pérez Terrane. In contrast, high grade metamorphic rocks are restricted to the Arroyo los Alamos Unit in the Piedra alta Terrane (Oyhantçabal et al., 2007), indicating a higher crustal level. A possibility to explain this difference could be to envisage local displacements along the Sarandí del Yí shear zone, which was certainly one of the main tectonic elements in the Ediacaran/Cambrian times (Oriolo et al., 2016b). It could also correspond to a pre-collision feature. The Rhyacian development of the bulk of the Piedra Alta Terrane possibly sets an upper time limit for the beginning of its behavior as a fault zone. From that time, the Sarandí del Yí shear zone was probably acting at successive pulses. Another speculative scenario could have been a thrust displacement of the Nico Pérez Terrane over the Piedra Alta Terrane, in which the Nico Pérez Terrane was uplifted and a deeper cortical level is now exposed at the present surface. This is supported by the recent work of Bologna et al. (2019) where the crust and upper mantle high-resistivity show no 
lithospheric-scale contrast across the Sarandí del Yí shear zone. This suggests local displacement, not affecting the upper mantle. Also, the preliminary results of Moho depth and $\mathrm{Vp} / \mathrm{Vs}$ ratio presented by Rodríguez et al. (2019) using one station near Tacuarembó (Nico Pérez Terrane) and the other near Salto city (Piedra Alta Terrane) show similar depth for the Moho.

Another important correlation between the Piedra Alta Terrane and the Nico Pérez Terrane could be the Statherian extensional tectonic event suggested by the emplacement of the Illescas rapakivi granite intruding the Valentines Block and the widespread Florida dike swarm occupying a great part of the Piedra Alta Terrane. Dyke swarms are distinctive representatives of mafic igneous activity associated with major continental rifting/plume events (e.g., Ernst and Buchan, 1997; Salminen et al., 2019) and are frequently related with rapakivi granites, particularly in Precambrian times. These dyke swarms associated with rapakivi granites, of similar ages, are an important tool for performing paleogeographic reconstructions of supercontinents. In Fact, rapakivi magmatism presents a characteristic temporal distribution in the world that could be contemporaneous with the supercontinent cycles (Larin, 2009; Salminen et al., 2019, and references therein).

The above mentioned evidence of reworking in the Nico Pérez Terrane could be indicative of a metacratonization in the sense of Abdelsalam et al. (2011), Liégeois et al. (2013), Santos et al. (2017, 2019) and Girelli et al. (2018), during the Neoproterozoic Brasiliano-Pan African orogenic cycle (Mallmann et al., 2007; Sánchez Bettucci et al., 2010b; Oriolo et al., 2017; among others).

\section{Conclusions}

The Nico Pérez Terrane is made up of a mosaic of tectonic blocks with different sizes, comprising a variety of rocks with different age and geological history. Archean granitoids are present in the Pavas Block, limited to the west by Cueva del Tigre shear zone. This block is affected by greenschist to amphibolite facies metamorphism, recording multiple metamorphic episodes and deformation. For the Valentines and Rivera blocks, a similar structural situation occurs, but the predominant rocks are Rhyacian granulites.
The Brasiliano-Pan African orogenic cycle, responsible for the amalgamation of Gondwana, affected the Nico Pérez Terrane and produced tectono-thermal activity and strong reworking of its rocks.

\section{Acknowledgements}

We are grateful to F. Santos Fernandes, V. Camara Maurer, E. Abelenda for the support in the laboratory and fieldwork and A. Latorres for the english review. Financial support was received from the Fundação de Amparo à Pesquisa do Estado de São Paulo (FAPESP) through grant 2013/12754-0 to UGC. We would like to thank $\mathrm{S}$. Oriolo and the anonymous reviewer for the rigorous and constructive comments and suggestions.

\section{References}

Abdelsalam, M.; Gao, S.; Liégeois, J.P. 2011. Upper mantle structure of the Saharan Metacraton. Journal of African Earth Sciences 60: 328-336. doi: 10.1016/j.jafrearsci.2011.03.009.

Almeida, F.F.M.; Amaral, G.; Cordani, U.G.; Kawashita, K. 1973. The Precambrian evolution of the South American cratonic margin, South of Amazona. In The Ocean Basins and Margins (Nairn, A.C.M.; Kanes, W.H.; Stehli, F.G.; editors). Plenum: 411-446. New York. doi: 10.1007/978-1-4684-3030-1_11.

Bologna, M.S.; Dragone, G.N.; Muzio, R.; Peel, E.; Núñez Demarco, P.; Ussami, N. 2019. Electrical structure of the lithosphere from Río de La Plata Craton to Paraná Basin: amalgamation of cratonic and re-fertilized lithospheres in SW Gondwanaland. Tectonics 38 (1): 77-94. doi: 10.1029/2018TC005148.

Bossi, J.; Umpierre, M. 1969. La petrología del yacimiento de hierro del arroyo Valentines, Florida: Uruguay. In Jornadas Argentinas de Geología, No. 4, Actas 1: 20 p. Mendoza.

Bossi, J.; Campal, N. 1987. Evidencias geológicas sobre la posible existencia de un núcleo cratónico de más de 2.000 Ma en el Noreste de Uruguay. In Simposio Sur-Brasilero de Geología, No. 3, Actas 2: 821-832. Curitiba.

Bossi, J.; Campal, N. 1992. Magmatismo y tectónica transcurrente durante el Paleozoico inferior del Uruguay. In Paleozoico Inferior de Ibero-América (Gutiérrez, J.; Saavedra, J.; Rábano, I.; editors). Universidad de Extremadura: 343-356. Alicante.

Bossi, J.; Gaucher, C. 2004. The Cuchilla Dionisio Terrane, Uruguay: An Allochthonous Block Accreted in the 
Cambrian to SW-Gondwana. Gondwana Research 7 (3): 661-674.

Bossi, J.; Fernández, A.; Elizalde, G. 1965. Predevoniano en el Uruguay. Boletín Facultad de Agronomía 78: 1-83.

Bossi, J.; Campal Gennari, N.; Preciozzi Porta, F.L. 1993. Predevoniano en el Uruguay: Parte I. Terreno Piedra Alta. Dirección Nacional de Minería y Geología: 50 p. Montevideo.

Bossi, J.; Campal Gennari, N.; Ferrando, L.A.; Gancio, F.; Schipilov, A.; Montaña, J.R.; Morales, H.L.; Piñeyro, D.; Sprechmann Heidenreich, P.W. 1998. Carta geológica del Uruguay: a escala 1/500.000 (CD-room). Universidad de la República, Facultad de Agronomía. Montevideo.

Brito Neves, B.B.; De Sá, J.M.; Nilson, A.A.; Botelho, N. 1995. A tafrogênese estateriana nos blocos paleoproterozóicos da América do Sul e processos suseqüentes. Geonomos 5 (3): 1-21.

Campal, N.; Schipilov, A. 1995. The Illescas bluish quartz rapakivi granite (Uruguay-South America): some geological features. In Symposium of Rapakivi Granites and Related rocks. Proceedings: 18-18. Belem.

Choubert, G.; Faure-Muret, A. 1969. Carte tectonique internationale de l'A frique, scale 1:5,000,000. Association of African Geological Surveys (ASGA) and United Nations Educational Scientific Surveys. Cultural Organisations (UNESCO): 9 p.

Cingolani, C.; Basei, M.; Bossi, J.; Piñeyro, D.; Uriz, N. 2012. U-Pb (LA-ICP-MS) Zircon Age of the La Paz Granite (Pando Belt, Uruguay): An Upper Neoproterozoic magmatic event in the Río de La Plata Craton, In South American Symposium on Isotope Geology, No. 8: p. 139. Medellín.

Cordani, U.G.; Soliani Jr., E. 1990. Idades K-Ar e Rb/Sr das "Ilhas Cristalinas" de Rivera e Aceguá (Uruguai e Rio Grande do Sul) e seu enquadramento geotectônico regional. Anais Academia Brasileira de Ciências 62:145-156.

Dinamige. 2016. Relevamiento Aerogeofísico de Magnetometría y Espectrometría de Rayos GammaMapa Geofísico del Uruguay. Ministerio de Industria, Energía y Minería. http://www.miem.gub.uy/mineriay-geologia/mapa-geofisico (last visited 08/05/2021).

Dragone, G.N.; Ussami, N.; Gimenez, M.E.; Lince Klinger, F.G.; Chaves, C.A.M. 2017. Western Parana suture/shear zone and the limits of Rio Apa, Rio Tebicuary and Rio de la Plata cratons from gravity data. Precambrian Research 291: 162-177. doi: 10.1016/j.precamres.2017.01.029.

Ellis de Luca, J.H. 1998. The precambrian supracrustal rocks of the Isla Cristalina de Rivera in northern Uruguay and their ore deposits. Ph.D. Thesis (Unpublished), University of Heidelberg: $196 \mathrm{p}$.

Ernst, R.E.; Buchan, K.L. 1997. Giant radiating dyke swarms: their use in identifying pre-Mesozoic large igneous provinces and mantle plumes. Geophysical Monograph, American Geophysical Union 100: 297 334. doi: 10.1029/GM100p0297.

Fragoso-César, A.R.S. 1980. O craton do Rio de la Plata e o cinturão Dom Feliciano no escudo Uruguaio-SulRiograndense. In Congresso Brasileiro de Geologia, Anais, No. 31, Actas: 2879-2882. Camboriú.

Franceschinis, P.R.; Rapalini, A.E.; Sánchez Bettucci, L.; Dopico, C.M.; Milanese, F.N. 2019. Paleomagnetic confirmation of the "unorthodox" configuration of Atlantica between 2.1 and 2.0 Ga. Precambrian Research 334 (105447). doi: 10.1016/j.precamres.2019.105447 .

Gaucher, C. 2000. Sedimentology, Paleontology and stratigraphy of the Arroyo del Soldado Group (Vendian to Cambrian, Uruguay). Beringeria 26: 1-120.

Gaucher, C.; Blanco, G. 2014. Batolito de Illescas. In Geología del Uruguay. Tomo 1: Predevónico (Bossi, J.; Gaucher, C.; editors). Universidad de la República: 209-214. Montevideo.

Gaucher, C.; Chemale Jr., F.; Bossi, J.; Castiglioni, E.A. 2010a. Grupo Cebollatí, Terreno Nico Pérez: definición y edad. In Congreso Uruguayo de Geología, No. 6. Actas en CD. Minas.

Gaucher, C.; Sial, A.N.; Halverson, G.F.; Frimmel, H.E. 2010b. The Neoproterozoic and Cambrian: a time of upheavals, extremes, and innovations. NeoproterozoicCambrian Tectonics, Global Change and Evolution: a focus on southwestern Gondwana. In Developments in Precambrian Geology (Gaucher, C.; Sial, A.N.; Halverson, G.P.; Frimmel, H.E.; editors): 16: 3-11.

Gaucher, C.; Frei, R.; Chemale, F.; Frei, D.; Bossi, J.; Martínez, G.; Chiglino, L.; Cernuschi, F. 2011. Mesoproterozoic evolution of the Río de la Plata Craton in Uruguay: at the heart of Rodinia? International Journal of Earth Sciences 100 (2-3): 273-288.

Gaucher, C.; Bossi, J.; Chemale Jr., F.; García, G.; Frei, R.; Frei, D. 2014a. Complejo La China: las rocas más antiguas del Uruguay. In Geología del Uruguay-Tomo 1: Predevónico (Bossi, J.; Gaucher, C.; editors). Universidad de la República: 141-154. Montevideo.

Gaucher, C.; Bossi, J.; Samaniego, L.; Frei, R. 2014 b. Complejo Tapes. In Geología del Uruguay-Tomo 1: Predevónico (Bossi, J.; Gaucher, C.; editors). Universidad de la República: 253-264. Montevideo.

Girelli, T.J.; Chemale Jr., F.; Lavina, E.L.C.; Laux, J.H.; Bongiolo, E.M.; Lana, C. 2018. Granulite accretion 
to Río de La Plata Craton, based on zircon U-Pb-Hf isotopes: Tectonic implications for Columbia Supercontinent reconstruction. Gondwana Research 56: 105-118. doi: 10.1016/j.gr.2017.12.010.

Gómez Rifas, C. 1989. Tectónica Cretácica en Uruguay. In Simposio Cretácico de América Latina, IGCP 242: A319-325. Buenos Aires.

Hartmann, L.A.; Leite, J.A.; McNaughton, N.J.; Santos, J.O.S. 1999. Deepest exposed crust of Brazil-SHRIMP establishes three events. Geology 27 (10): 947-950. doi:10.1130/0091-7613(1999)027<0947:DECOBS $>2.3 . \mathrm{CO} ; 2$.

Hartmann, L.A.; Campal, N.; Santos, J.O.S.; Mcnaughton, N.J.; Bossi, J.; Schipilov, A.; Lafon, J.M. 2001. Archean crust in the Río de La Plata craton, Uruguay-SHRIMP $\mathrm{U}-\mathrm{Pb}$ zircon reconnaissance geochronology. Journal of South American Earth Sciences 14: 557-570. doi: 10.1016/S0895-9811(01)00055-4.

Hurley, P.M.; De Almeida, F.F.M.; Melcher, G.C.; Cordani,U.G.; Rand, J.R.; Kawashita, K.; Vandoros, P.; Pinson Jr., W.H.; Fairbairn, H.W. 1967. Test of continental drift by comparison of radiometric ages. Science 157 (3788): 495-500. doi: 10.1126/science.157.3788.495.

Larin, A.M. 2009. Rapakivi granites in the geological history of the earth. In Part 1, magmatic associations with rapakivi granites: age, geochemistry, and tectonic setting. Stratigraphy and Geological Correlation 17 (3): 235. doi: 10.1134/S0869593809030010.

Liégeois, J.P.; Abdelsalam, M.G.; Ennih, N.; Ouabadi, A. 2013. Metacraton: nature, genesis and behavior. Gondwana Research 23 (1): 220-237. doi: 10.1016/j.gr.2012.02.016.

Lossada, A.C.; Rapalini, A.E.; Sánchez Bettucci, L. 2014. Enjambre de diques básicos de Nico Pérez-Zapicán, Uruguay: evidencias radimétricas y paleomagnéticas sobre su edad. Revista de la Asociación Geológica Argentina 71 (3): 345-355.

Loureiro, J. 2008. Mapa Geológico de la Isla Cristalina De Rivera. Internal report (Unpublished), Dirección Nacional de Minería y Geología: 1 p. Montevideo.

Loureiro, J.; Silva, H.; Sánchez Bettucci, L. 2019. Mapa Geológico: El Arqueano en el Uruguay. Revista Investigaciones 2 (1): 28-35. Montevideo. https://www. miem.gub.uy/sites/default/files/a3_loureiro_arqueano. pdf (last visited 10/05/2021).

Ludwig, K.R. 2009. SQUID 2: a user's manual. Berkeley Geochronology Center. Special Publication 5: $1-110$.

Mallmann, G.; Chemale Jr., F.; Ávila, J.N.; Kawashita, K.; Armstrong, R.A. 2007. Isotope geochemistry and geochronology of the Nico Pérez Terrane, Río de La
Plata Craton, Uruguay. Gondwana Research 12: 489508. doi: 10.1016/j.gr.2007.01.002.

Masquelin, H. 2006. El escudo uruguayo. In Cuencas Sedimentarias de Uruguay, geología, paleontología y recursos minerales: Paleozoico(Ubilla, M.; Veroslavsky, G.; editors). Facultad de Ciencias, Dirac 3: 37-106. Montevideo.

Masquelin, H.; Silva, H.; Sánchez Bettucci, L.; Núñez, P.; Pascual, S.; Muzio, R.; Peel, E.; Scaglia, F. 2017. Lithologies, structure and basement cover relationships in the schist belt of the Dom Feliciano Belt in Uruguay. Brazilian Journal of Geology 47 (1): 21-42. doi: 10.1590/2317-4889201720160119.

Massonne, H.J.; Tikovsky, T.; Hartmann, L.A. 2001. Petrology of the $2.0 \mathrm{Ga}$ old Santa Maria Chico granulites in southern Rio Grande do Sul, Brazil, and implications for crustal thickening in Paleoproterozoic times. In Congreso Latinoamericano de Geología, No. 11, Actas en CD. Montevideo.

Montaña, J.; Sprechmann, P. 1993. Calizas estromatolíticas y oolíticas en el Uruguay y definición de la Formación Arroyo de la Pedrera (Cámbrico medioCaradociano). In Simposio Internacional del NeoproterozoicoCámbrico de la Cuenca del Plata, No. 1, Actas 2: p. 53.

Oriolo, S.; Oyhantçabal, P.; Basei, M.A.S.; Wemmer, K.; Siegesmund, S. 2016a. The Nico Pérez Terrane (Uruguay): From Archean crustal growth and connections with the Congo Craton to late Neoproterozoic accretion to the Río de La Plata Craton. Precambrian Research 280: 147-160. doi: 10.1016/j.precamres.2016.04.014.

Oriolo, S.; Oyhantçabal, P.; Wemmer, K.; Basei, M.A.S.; Benowitz, J.; Pfänder, J.; Hannich, F.; Siegesmund, S. 2016b. Timing of deformation in the Sarandí del Yí Shear Zone, Uruguay: Implications for the amalgamation of western Gondwana during the Neoproterozoic Brasiliano-Pan-African Orogeny. Tectonics 35: 754-771. doi: 10.1002/2015TC004052.

Oriolo, S.; Oyhantçabal, P.; Wemmer, K.; Siegesmund, S. 2017. Contemporaneous assembly of Western Gondwana and final Rodinia break-up: implications for the supercontinent cycle. Geoscience Frontiers 8 (6): 1431-1445.

Oriolo, S.; Oyhantçabal, P.; Konopásek, J.; Basei, M.A.; Frei, R.; Sláma, J.; Wemmer, K.; Siegesmund, S. 2019. Late Paleoproterozoic and Mesoproterozoic magmatism of the Nico Pérez Terrane (Uruguay): Tightening up correlations in southwestern Gondwana. Precambrian Research 327: 296-313. doi: 10.1016/j.precamres.2019.04.012. 
Oyhantçabal, P.; Muzio, R.; De Souza, S. 1993. Geología y aspectos estructurales del borde orogénico en el extremo sur del cinturón Dom Feliciano. Revista Brasileira de Geociências 23 (3): 296-300.

Oyhantçabal, P.; Spoturno, J.; Loureiro, J. 2007. Caracterización geológica de las rocas Paleoproterozoicas de la región Centro-Sur del Uruguay (Terreno Piedra Alta-Cratón del Río de La Plata). In Congreso Uruguayo de Geología, No. 5, Actas en CD. Montevideo.

Oyhantçabal, P.; Siegesmund, S.; Wemmer, K. 2011. The Río de la Plata Craton: a review of units, boundaries, ages, and isotopic signature. International Journal Earth Science (Geol Rundsch) 100: 201-220. doi: 10.1007/s00531-010-0580-8.

Oyhantçabal, P.; Wagner-Eimer, M.; Wemmer, K.; Schulz, B.; Frei, R.; Siegesmund, S. 2012. Paleo- and Neoproterozoic magmatic and tectonometamorphic evolution of the Isla Cristalina de Rivera (Nico Pérez Terrane, Uruguay). Journal of South American Earth Sciences 101: 1745-1762. doi: 10.1007/s00531-012-0757-4.

Oyhantçabal, P.; Oriolo, S.; Philipp, R.P.; Wemmer, K.; Siegesmund, S. 2018. The Nico Pérez Terrane of Uruguay and Southeastern Brazil. In Geology of Southwest Gondwana (Siegesmund, S.; Basei, M.; Oyhantçabal, P.; Oriolo, S.; editors). Regional Geology Reviews, Springer: 161-188. doi: 10.1007/978-3-319-68920-3_7.

Preciozzi, F.; Spoturno, J.; Heinzen, W. 1979. Carta geoestructural del Uruguay, escala 1/2.000.000. Ministerio de Industria y Energía, Instituto Geológico Ingeniero Eduardo Terra Arocena: 1-62. Montevideo.

Preciozzi, F.; Spoturno, J.; Heinzen, W.; Rossi, P. 1985. Carta Geológica del Uruguay a escala 1:500.000. Texto explicativo. Ministerio de Industria y Energía, Dirección Nacional de Minería y Geología: 1-92. Montevideo.

Rapela, C.W.; Pankhurst, R.J.; Casquet, C.; Fanning, C.M.; Baldo, E.G.; González-Casado, J.M.; Galindo, C.; Dahlquist, J. 2007. The Río de la Plata Craton and the assembly of SW Gondwana. Earth-Science Reviews 83: 49-82

Rodríguez, M.; Curbelo, A.; Castro, H.; Dell'Acqua, D.; Latorres, E.; Sánchez Bettucci, L.; Assumpção, M. 2019. Crustal thickness and vp/vs ratio for three stations in Uruguay using receiver function analysis: preliminary results. In Simpósio Brasileiro de Sismologia, No. 3: p. 87. San Pablo.
Salminen, J.; Klein, R.; Mertanen, S. 2019. New rock magnetic and paleomagnetic results for the $1.64 \mathrm{Ga}$ Suomenniemi dyke swarm, SE Finland. Precambrian Research 329: 195-210. doi: 10.1016/j.precamres.2018.01.001.

Sánchez Bettucci, L.; Peel, E.; Oyhantçabal, P. 2010a. Precambrian geotectonic units of the Río de La Plata craton. International Geology Review 52 (1): 32-50. doi: 10.1080/00206810903211104.

Sánchez Bettucci, L.; Peel, E.; Masquelin, H. 2010 b. Neoproterozoic tectonic synthesis of Uruguay. International Geology Review 52 (1): 51-78. doi: 10.1080/00206810903358095.

Santos, J.O.S.; Hartmann, L.A.; Bossi, J.; Campal, N.; Schipilov, A.; Piñeyro, D.; Mcnaughton, N.J. 2003. Duration of the Trans-Amazonian Cycle and its correlation within South America based on U-Pb SHRIMP geochronology of the La Plata Craton, Uruguay. International Geology Review 45: 27-48. doi: 10.2747/0020-6814.45.1.27.

Santos, J.O.S.; Chernicoff, C.J.; Zappettini, E.O.; McNaughton, N.J.; Greau, Y. 2017. U-Pb geochronology of Martín García, Sola, and Dos Hermanas Islands (Argentina and Uruguay): Unveiling Rhyacian, Statherian, Ectasian, and Stenian of a forgotten area of the Río de la Plata Craton. Journal of South American Earth Sciences 80: 207-228.

Santos, J.O.S.; Chernicoff, C.J.; Zappettini, E.O.; Mcnaughton, N.J.; Hartmann, L.A. 2019. Large geographic and temporal extensions of the Río de La Plata Craton, South America and its metacratonic eastern margin. International Geology Review 61 (1): 56-85. doi: 10.1080/00206814.2017.1405747.

Sato, K.; Tassinari, C.; Basei, M.A.S.; Júnior, O.; Onoe, A.; Souza, M. 2014. Sensitive High Resolution Ion Microprobe (SHRIMP IIe/MC) of the Institute of Geosciences of the University of São Paulo, Brazil: Analytical method and first results. Geologia USP. Série Científica 14: 3-18. doi: 10.5327/Z1519-874X201400030001.

Soliani, E. 1986. Os dados geocronológicos do Escudo Sul-Rio Grandense e suas implicaçoes de ordem geotectônica. Ph.D. Thesis (Unpublished), Universidade de São Paulo: 243 p.

Williams, I.S. 1998. U-Th-Pb geochronology by ion microprobe. Reviews in Economic Geology 7: 1-35. doi: 10.5382/Rev.07.01. 